\title{
Mersin-Yumuktepe. Une mise au point sur les derniers travaux
}

Isabella Caneva et Éric Jean

\section{Q OpenEdition}

10 Journals

Édition électronique

URL : http://journals.openedition.org/anatoliaantiqua/368

DOI : 10.4000/anatoliaantiqua.368

Éditeur

IFEA

Édition imprimée

Date de publication : 1 mai 2016

Pagination : 13-34

ISBN : 9782362450648

ISSN : 1018-1946

Référence électronique

Isabella Caneva et Éric Jean, « Mersin-Yumuktepe. Une mise au point sur les derniers travaux»,

Anatolia Antiqua [En ligne], XXIV | 2016, mis en ligne le 11 décembre 2018, consulté le 16 février 2021.

URL : http://journals.openedition.org/anatoliaantiqua/368 ; DOI : https://doi.org/10.4000/

anatoliaantiqua. 368 


\section{ANATOLIA ANTIQUA ESKI ANADOLU}

\section{XXIV}

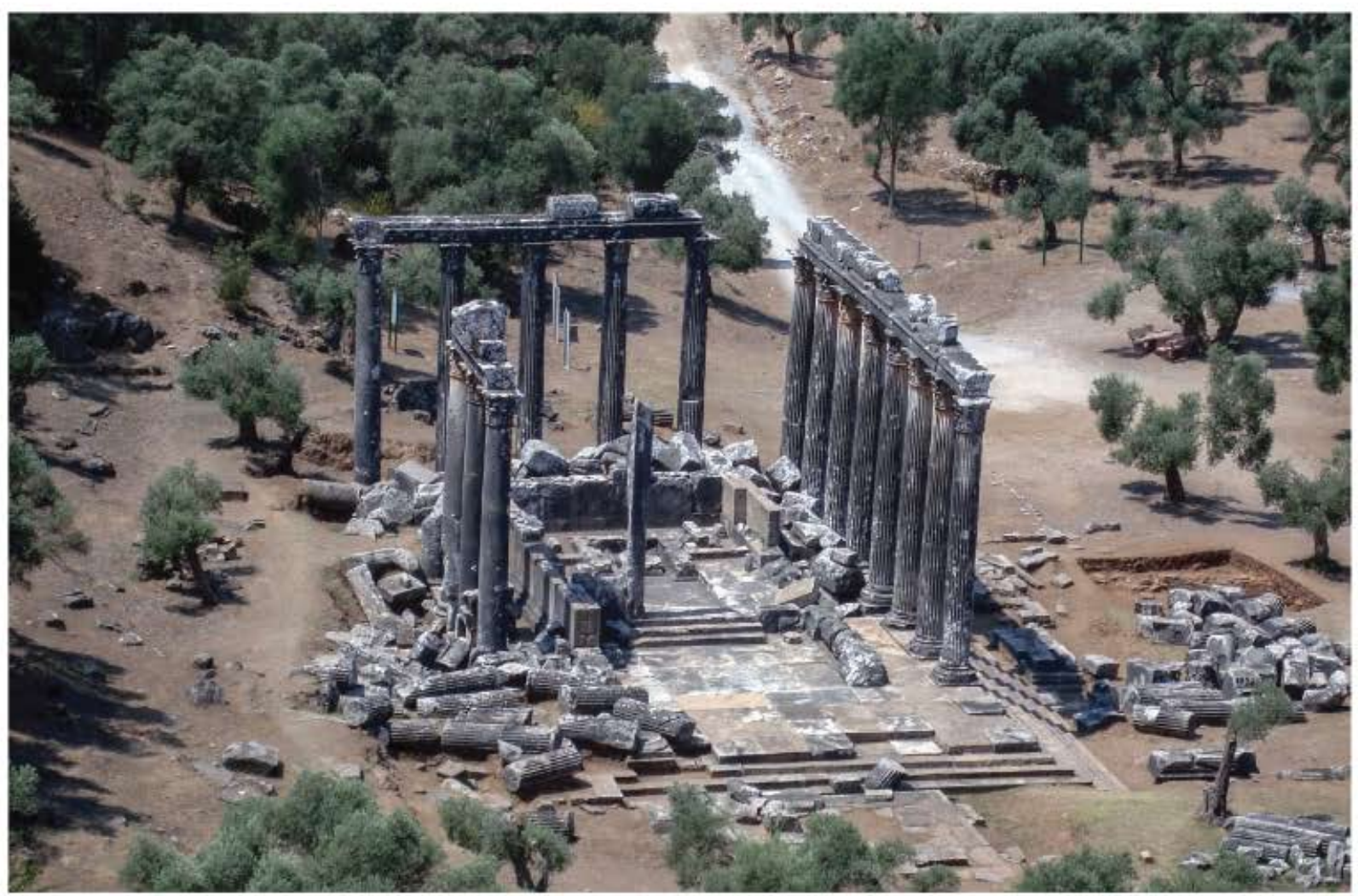

INSTITUT FRANÇAIS D'ETUDES ANATOLIENNES GEORGES-DUMEZIL

CNRS USR 3131

DE BOCCARD 


\section{TABLE DES MATIERES}

Ergul KODAS,

Le surmodelage du crâne au Néolithique au Proche-Orient : techniques de surmodelage et

expérimentations

Isabella CANEVA et Eric JEAN,

Mersin-Yumuktepe : une mise au point sur les derniers travaux

Turan EFE et Bérengère PERELLO,

Second Millenium site distribution and pottery of Inland Northwestern Anatolia

Antoine PEREZ,

Amida 6 : Antiochos IV, le "Hanigalbat" et la Sophène

Ergün LAFLI et Hadrien BRU,

Inscriptions et monuments funéraires gréco-romains d'Anatolie occidentale

Oğuz TEKİN et Aliye EROL-ÖZDİZBAY,

Coins from Allianoi excavations: Campaign of 2001

Nuran ŞAHIN,

Etude iconographique des monnaies autonomes frappées par Colophon-sur-Mer :

nouveaux acquis

Vera SAUER,

Konventionelle Individualität. Zur Münzprägung nordanatolischer Städte in der römischen

Kaiserzeit

Oğuz TEKIN,

Balance weights in the collection of the Anatolian Civilizations Museum in Ankara

\section{CHRONIQUES DES TRAVAUX ARCHEOLOGIQUES EN TURQUIE 2015}

Çiğdem MANER,

Preliminary report on the third season of the Konya-Ereğli Survey (KEYAR) 2015

Dominique BEYER, Isabelle CHALIER et Françoise KIRNER,

Rapport préliminaire sur les travaux de la mission archéologique de Zeyve Höyük-Porsuk 2015 ...

Sami PATACI et Ergün LAFLI,

Field surveys in Ardahan in 2015

Jean-Charles MORETTI avec la collaboration de Nicolas BRESCH, Isabel BONORA,

Jean-Jacques MALMARY et Olivier RISS,

Claros, le temple d'Apollon : travaux réalisés en 2015

Serdar Hakan ÖZTANER,

La basilique civile de Nysa du Méandre

Abuzer KIZIL, Koray KONUK, Patrice BRUN, Laurent CAPDETREY, Raymond DESCAT,

Pierre FROHLICH, Didier LAROCHE, Enora LE QUERE, Francis PROST, Baptiste VERGNAUD,

Eurômos : rapport préliminaire sur les travaux réalisés en 2015

O. HENRY et E. ANDERSON, Chr. BOST, Ö. ÇAKMAKLI, F. CEDERLING, A. COMMITO,

M. CORMIER-HUGUET, A. COUTELAS, A. DOLEA, D. ERGENÇ, A. FRECCERO,

A. FREJMAN, P. LEBOUTEILLER, F. LESGUER, D. LÖWENBORG, V. LUNGU,

Fr. MARCHAND-BEAULIEU, A. SITZ, P. DE STAEBLER, B. VERGNAUD, 


\section{MERSIN-YUMUKTEPE UNE MISE AU POINT SUR LES DERNIERS TRAVAUX}

\section{INTRODUCTION}

A quatre-vingts ans de la découverte scientifique du site de Mersin-Yumuktepe, nous avons pensé qu'il était temps de faire le point sur les travaux archéologiques effectués jusqu'à présent. Le site de Yumuktepe présente à la fois l'inconvénient et l'avantage de pouvoir relier les données des nouvelles fouilles à celles des anciennes, ce qui permet de réévaluer et de compléter réciproquement les résultats. C'est pourquoi, après un bref historique des recherches et des objectifs et méthodes des différentes générations de fouilles ${ }^{1}$, les travaux effectués ces dernières années seront ici résumés. A partir de cette révision, une nouvelle perspective et une réflexion sur les objectifs à venir seront envisagées.

Yumuktepe est un grand höyük, qui couvre près de $5 \mathrm{ha}$, son sommet formant un petit plateau irrégulier de 1 ha environ. De forme ovale et orienté Sud-Est/Nord-Ouest, son diamètre à la base est de $300 \mathrm{~m}$ et son sommet s'élève à $23 \mathrm{~m}$ au-dessus de la plaine ( $25 \mathrm{~m}$ à l'époque des premières fouilles dans les années 30 ) et $50 \mathrm{~m}$ au-dessus du niveau de la mer.

D'après la longue stratigraphie du site, la position de Mersin-Yumuktepe revêtait certainement un intérêt stratégique dans toutes les périodes qui y sont représentées. Calé entre les montagnes et la mer, et pourtant lié par de nombreuses voies de communication au plateau anatolien et ouvert sur la Méditerranée avec sont port, il fut comparé à une sentinelle montant la garde entre la Cilicie plane, dont il occupe l'extrémité occidentale, et la Cilicie trachée 2 .
Situé à $2 \mathrm{~km}$ du centre de la ville moderne de Mersin, complètement entouré par de nouveaux quartiers, le site montre encore aujourd'hui la persistance du succès de sa position! (Fig. 1)

\section{HISTORIQUE DES RECHERCHES}

$\mathrm{Au} 20^{\mathrm{e}}$ siècle, longtemps après la dernière occupation du site, diverses activités humaines endommagèrent fortement le höyük. Plusieurs fosses y furent retrouvées, creusées par des soldats d'une garnison française installée sur son sommet aux lendemains de la Grande Guerre. Au début des années 40, les pierres de la "fortification hittite", laissées en place après la campagne de 1939 dans l'espoir d'une future restauration, furent utilisées pour la construction d'un chemin de fer. La morphologie même du site fut transformée à partir de 1963, quand le höyük fut aménagé en parc public. Des travaux de terrassements y furent effectués, des arbres plantés, puis, au fil des ans, des installations modernes construites : un escalier monumental de la base au sommet, des murs de soutènement en pierres, des canalisations, des poteaux électriques, des toilettes, etc. Le sommet était devenu une large surface plane, la pente fut coupée en terrasses et d'épaisses couches de terre furent accumulées sur les versants du höyük qui changea ainsi radicalement de forme ${ }^{3}$.

Longtemps appelé Yümüktepe, le site de Mersin-Yumuktepe est souvent abrégé en Mersin, mais prend aussi parfois le nom de Mersin-Soğuksutepe, du nom de la rivière Soğuksu ("eau froide"), sur la rive orientale de laquelle il est situé4.

*) Università del Salento, Dipartimento di Beni Culturali, Lecce, Italie.

**) Hitit Üniversitesi, Fen-Edebiyat Fakültesi, Arkeoloji Bölümü, Çorum, Turquie.

1) Garstang 1953 ; Köroğlu, K. 1998 ; Caneva et Sevin 2004 ; Caneva et Köroğlu, G. 2010.

2) Garstang $1953: 3$.

3) Le secteur où avait été dégagé le niveau XXVI, par exemple, et laissé tel quel à la fin des fouilles de Garstang, fut redécouvert sous $5 \mathrm{~m}$ de remblais par la nouvelle équipe (Caneva et Marcolongo 2004 : 26 ; Caneva 2010a).

4) Le nom Yumuk Tepe vient du turc "yumuk" qui signifie "doux" ou "fermé". Le nom "Yümük Tepe", donné par erreur par les archéologues britanniques, n'a aucun sens en turc (Sevin 2004 : 19 n. 1). Quant à la rivière Soğuksu, elle est aussi nommée Müftü Deresi ("ruisseau du mufti") ou Efrenk Çayı (Sevin 2004 : 16) en référence à l'occupation française. 


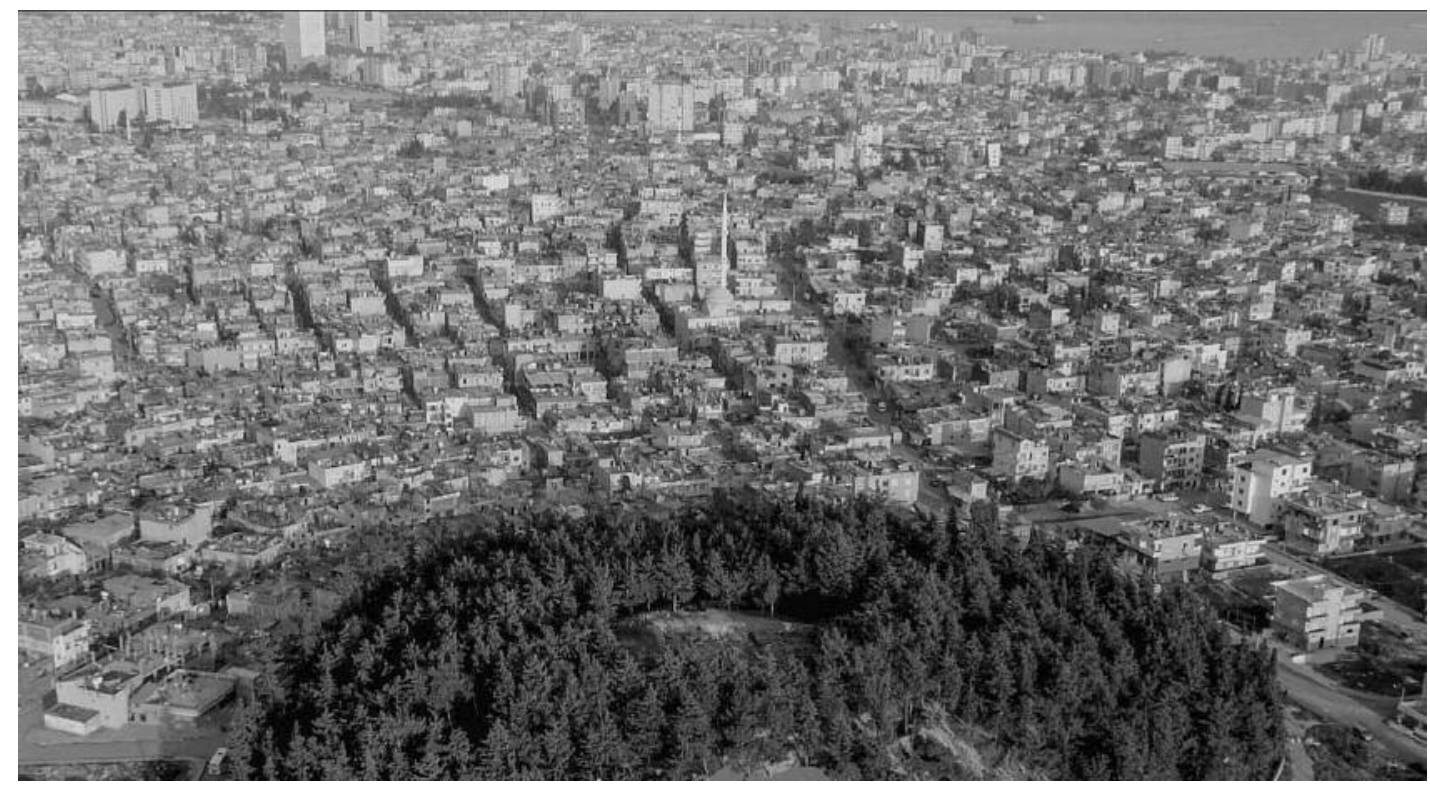

Fig. 1 : Le site de Yumuktepe à Mersin : du village néolithique à la métropole moderne.

Le site était probablement à l'origine un véritable site côtier (pendant la période néolithique, du moins), situé près de l'embouchure du Soğuksu ${ }^{5}$, et ce dernier coulait au moins cent mètres à l'Est du höyük pendant toutes les périodes d'occupation du site. C'est vraisemblablement au Moyen-Age que le Soğuksu changea de cours et provoqua l'abandon du site et la perte d'une portion majeure de sa partie occidentale ${ }^{6}$.

Les différentes stratégies d'installations humaines modifièrent graduellement la formation et la topographie du site, la taille, la localisation et la disposition fonctionnelle des divers habitats variant avec les périodes ${ }^{7}$. En outre, les fouilles récentes ont montré que l'installation des occupants du Chalcolithique s'étendait aussi à côté de la base du höyük au Sud, dans une zone vierge de vestiges néolithiques, donnant ainsi naissance à une seconde zone d'accumulations de dépôts archéologiques et donc à plusieurs stratigraphies parallèles et décalées. Incomprises par Garstang, les discontinuités topographiques et stratigraphiques du tell indiquent, en effet, que la première occupation néolithique était beaucoup plus réduite que le périmètre actuel du site au pied du höyük et que plusieurs secteurs, à altitudes identiques, avaient été formés à différentes périodes. Ainsi, les niveaux Bronze Récent du chantier sud se trouventils à quelque quatre mètres plus bas que leurs contreparties au Nord-Ouest, à des altitudes globalement semblables à celles des niveaux du Chalcolithique Récent ${ }^{8}$. Or, le remblai découvert dans le chantier sud entre les niveaux "hittite ancien" et "récent" a une épaisseur de près de quatre mètres et pourrait correspondre au comblement délibéré du décalage entre les altitudes des deux zones d'occupation.

Mersin-Yumuktepe connut deux générations de fouilles, la première ayant commencé dans les années 1930, peu après celles de Tarse. Une réévaluation de la stratigraphie fut en outre effectuée, sans fouilles, entre les deux projets, pour ce qui concerne les niveaux néolithiques et chalcolithiques ${ }^{9}$.

C'est dans le cadre d'une prospection menée dans la région en 1936 par l'expédition Neilson et dirigée par John Garstang que le site de Yumuktepe fut choisi pour y entreprendre des fouilles archéologiques ; des sondages y furent effectués pendant l'hiver 1936-1937 ${ }^{10}$. Après trois missions de fouilles, menées pendant les hivers 1937-1938, 1938-1939

5) Sevin et Caneva $1998: 5$.

6) Caneva et Marcolongo 2004 : 26, fig. 2 ; Caneva 2010a.

7) Caneva et Marcolongo $2004: 30$.

8) Caneva et Marcolongo $2004: 28$; Sevin et Köroğlu, K. $2004: 80$.

9) Breniquet 1995.

10) La prospection de la Neilson Expedition fut complétée par des sondages archéologiques effectués sur les sites de Çavuşlu Höyük, Kazanlı Höyük, Sirkeli Höyük et Mersin-Yumuktepe (Garstang 1937). 
et 1939-1940, les recherches durent être interrompues peu après le début de la Seconde Guerre mondiale. Au lendemain de la guerre, une dernière saison de fouille eut lieu pendant l'hiver 1946-1947'11. Rappelons le nom de quelques grandes personnalités du monde de la recherche qui participèrent peu ou prou à ces fouilles britanniques : Seton H.F. Lloyd, Richard D. Barnett, Veronica Seton-Williams, Oliver Gurney, Vere Gordon Childe, Miles Burkitt et William Brice. C'est la découverte de fragments d'outils en obsidienne et de Black Burnished Ware dans le lit du Soğuksu qui incita l'archéologue britannique à choisir ce site comme lieu de fouilles systématiques. C'est donc le Néolithique qui avait attiré son attention et probablement amené à la constitution d'une équipe essentiellement composée de préhistoriens. Enseignant les méthodes et pratiques de l'archéologie à l'Université de Liverpool, John Garstang y mena l'une des fouilles les plus modernes de son époque, soumettant en outre des échantillons botaniques, de céramique et d'objets en pierre et en métal à des analyses de laboratoire. Garstang fut précisément l'un des tout premiers à avoir utilisé le sondage stratigraphique, méthode qu'il avait expérimentée à Jéricho. Grâce à lui, le site devint la plus longue et la plus fiable des références stratigraphiques, qui permit de mettre en corrélation toute la séquence des cultures d'Anatolie occidentale et centrale avec la chronologie syro-mésopotamienne.

Garstang choisit de concentrer ses recherches dans la partie nord occidentale, qui lui semblait particulièrement importante par le fait qu'elle dominait la rivière Soğuksu et parce que son versant était le plus escarpé du site et présentait un profil largement entaillé par l'érosion fluviale. Deux chantiers y furent donc ouverts : celui du sommet ou "Main Area", couvrant une superficie d'environ 0,5 ha, et le sondage stratigraphique "X", de 8 x $15 \mathrm{~m}$, ouvert depuis l'extrémité nord du chantier principal et suivant la pente vers l'Ouest-Nord-Ouest. Quelques autres petits sondages furent effectués, notamment le sondage "A" sur le versant occidental du site, et concernèrent essentiellement des phases du Néolithique Ancien. Sur le sommet du höyük, dans la partie méridionale du chantier principal, une pierre carrée marquait l'altitude de $+25 \mathrm{~m}$ par rapport au point zéro de référence topographique fixé dans le lit de la rivière.
Concernant la complexité de la stratigraphie du site, le système d'installation des occupants sur des terrasses, relativement bien compris par Garstang pour ce qui est des niveaux du deuxième millénaire ${ }^{12}$, ne le fut pas pour les niveaux antérieurs. Cette difficulté, rencontrée ailleurs dans les fouilles de höyük, ne put être ultérieurement contrebalancée à Yumuktepe par la vérification systématique des enregistrements effectués par les fouilleurs, depuis que les dessins originaux - archivés en Angleterre - furent détruits dans une attaque aérienne pendant la Seconde Guerre mondiale. C'est pourquoi Garstang, pour la publication finale, répéta souvent verbatim les rapports préliminaires et se fonda sur les carnets et croquis de fouilles, ce qui provoqua des différences d'échelle et beaucoup d'approximation dans les illustrations ${ }^{13}$. Par ailleurs, en dehors des chantiers nord-ouest, aucune autre zone du site ne fut fouillée et, dans la publication, une grande partie du matériel n'est que très peu décrite et documentée, notamment pour ce qui concerne les restes botaniques, zoologiques et anthropologiques ${ }^{14}$.

La reprise des activités archéologiques à Yumuktepe était donc perçue comme une nécessité.

\section{OBJECTIFS ET METHODES : PREMIERS RESULTATS DES NOUVELLES FOUILLES}

Près de cinquante ans après la dernière campagne de Garstang, les fouilles à Yumuktepe reprirent en 1993 sous la direction de Veli Sevin, de l'Université d'Istanbul, et d'Isabella Caneva, de l'Université La Sapienza à Rome, puis de Lecce, en Italie, et qui en assume la direction depuis 2001.

Respectivement préhistorienne et protohistorien de formation, Isabella Caneva et Veli Sevin s'étaient fixés des objectifs spécifiques. Parmi ceux-ci, la reconnaissance stratigraphique de tous les niveaux d'occupation de Yumuktepe pour reconstruire les dynamiques de formation du dépôt archéologique, d'où la nécessité d'une plus grande diversification des zones étudiées, une exploration qui atteigne le sol vierge, l'investigation planimétrique de chaque niveau architectural, l'étude de l'intensité et de la répartition géographique des différents niveaux, de façon à comprendre pourquoi des niveaux n'étaient pas représentés ou étaient sous-représentés ${ }^{15}$. Se

11) Brice 2004.

12) Caneva et Marcolongo $2004: 29$

13) Garstang 1953 : vi.

14) La mort de sa femme Peggy, en 1949, avait jeté Garstang dans un état dépressif qui explique en grande partie le caractère insatisfaisant de la publication (Sevin 2004 : 16). En 1956, à l'âge de 80 ans, conférencier dans une croisière en Méditerranée qui fit escale à Mersin, une fois qu'il y eut grimpé avec une aide, il présenta le site de manière monumentale aux participants, et mourut la nuit suivante dans le bateau en route pour Beyrouth (Brice $2004: 7$ ).

15) Sevin $2004: 18$. 


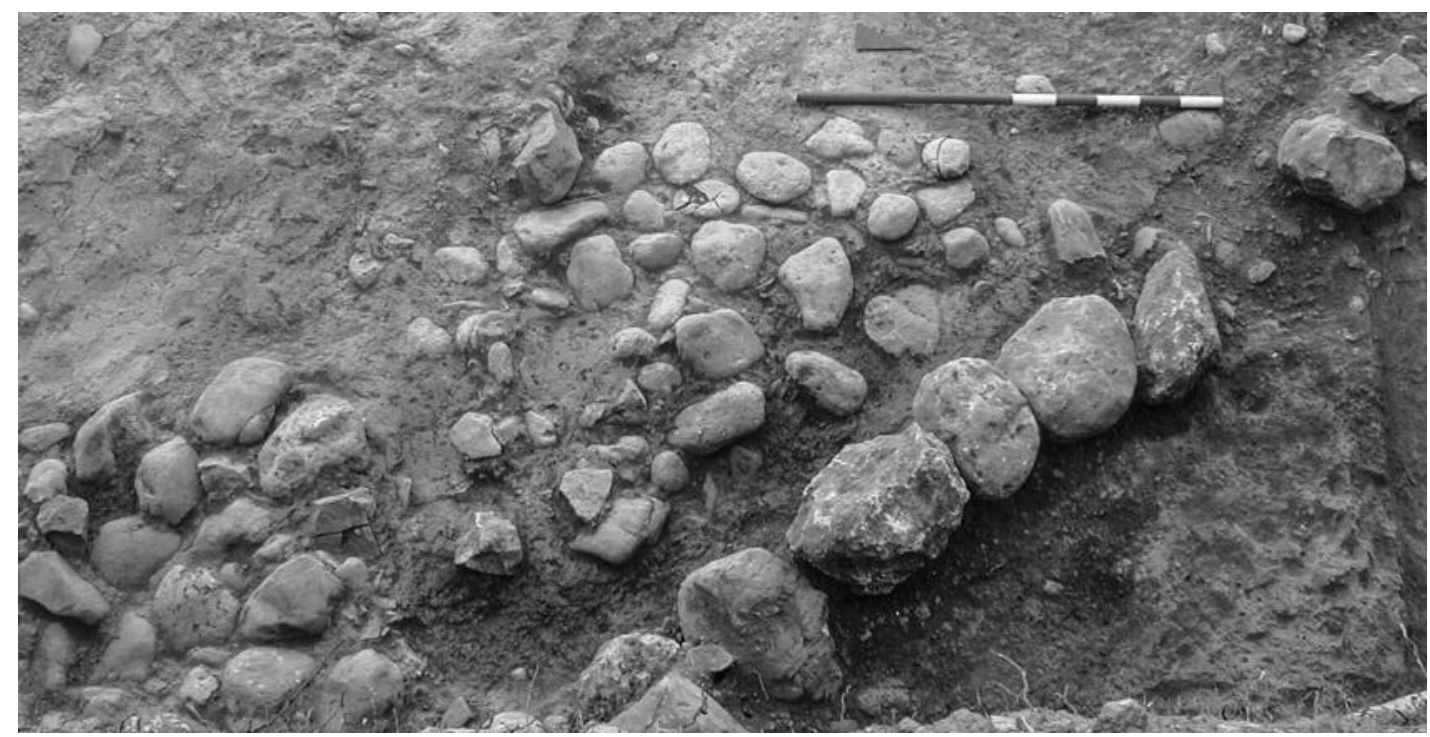

Fig. 2 : Le bord de la tranchée "X" retrouvé dans les dépôts néolithiques.

concentrant à nouveau sur le secteur nord-occidental pour l'étude des niveaux les plus anciens (Néolithique, Chalcolithique et Bronze Ancien) et sur le sommet du tell pour les niveaux les plus récents (âge du Fer et Moyen-Age), la nouvelle équipe s'ouvra aussi peu à peu aux secteurs sud et, très partiellement, est du site. Sur le versant méridional, une fouille en escalier fut ouverte en 1997 pour vérifier l'hypothèse des formations stratigraphiques décalées dans la partie méridionale du tell. A partir de 2006, à une profondeur de $5 \mathrm{~m}$ environ, elle fut élargie sur les deux côtés, passant de 5 à $14 \mathrm{~m}$.

Les transformations récentes du site, évoquées plus haut, en avaient fait disparaître les derniers vestiges, ainsi que les points de référence topographique (le point zéro et l'altitude maximale). Le premier défi fut donc d'établir la correspondance entre les découvertes des nouvelles fouilles et celles des anciennes. Le chantier " $X$ " de la première génération de fouilles ne fut localisé sur le terrain qu'en 2002, et que dans la partie la plus septentrionale de la fouille (secteur C6 : Fig. 2) ${ }^{16}$. Un second problème tenait à la confiance à accorder aux résultats de fouilles effectuées dans les nouveaux chantiers sud et est du fait de leur étroitesse sur la pente de la colline, où la dynamique de terrassement est plus active et peut être plus difficile à comprendre. Dans ce cadre, les nouvelles fouilles permirent, jusqu'à présent, de mettre en évidence quatre grandes périodes de construction de terrasses. La première, mise en lumière au Nord-Ouest, date du Néolithique Récent (niveaux XXV-XXIV); la seconde, toujours au Nord-Ouest, du Chalcolithique Moyen, le niveau XVI étant représenté de la citadelle au sommet de l'époque jusqu'au niveau de la rivière actuelle (Fig. 3) ; la troisième période est celle des niveaux de l'âge du Bronze ; la quatrième, enfin, est la période byzantine ${ }^{17}$. En outre, un petit sondage effectué au tout début des nouvelles fouilles au pied du höyük dans la partie méridionale révéla aussi l'absence de vestiges néolithiques et la présence de niveaux probablement attribuables au Chalcolithique Récent directement sur le sol vierge, suggérant donc la présence d'une cinquième période d'habitats en terrasses.

Le site fut occupé de façon quasi ininterrompue du Néolithique Ancien (7000 av. J.-C.) à l'époque byzantine (XIII ${ }^{\mathrm{e}}$ siècle ap. J.-C.). Une interruption majeure est toutefois toujours suspectée pendant le quatrième millénaire avant notre ère, entre le Chalcolithique Récent (LC 2) et le Bronze Ancien (Fig. 4).

Dans le secteur nord-ouest, après avoir repris et poursuivi en profondeur le sondage "A" de Garstang, la fouille fut élargie vers le $\operatorname{Sud}^{18}$. De nouvelles phases de construction furent ainsi repérées, qui reculèrent la date des plus anciennes cultures à Yumuktepe, tout en confirmant l'absence de niveaux acéramiques, les premiers occupants du site s'étant installés sur une dune marine fossile. Les plus an-

16) Avant cela, la seule trace des anciennes fouilles à avoir été en partie préservée était le profond sondage "A", qui avait été effectué dans la coupe faite par la rivière dans la partie occidentale du site.

17) Caneva et Marcolongo 2004 : 29-30.

18) Caneva 2004a : 33, fig. 2. 


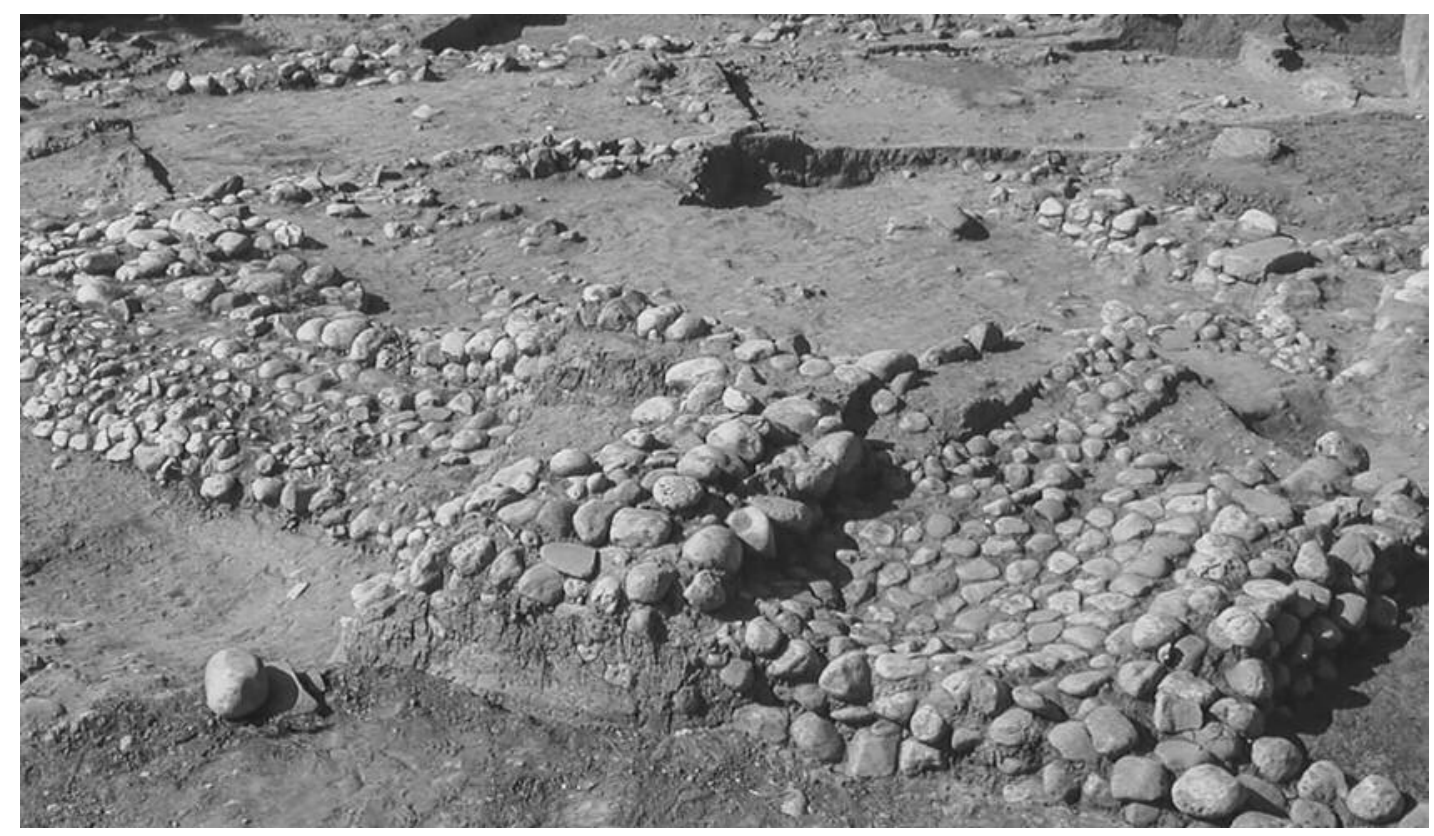

Fig. 3 : Les maisons en terrasses du Chalcolithique Moyen (au fond) creusées dans les niveaux néolithiques.
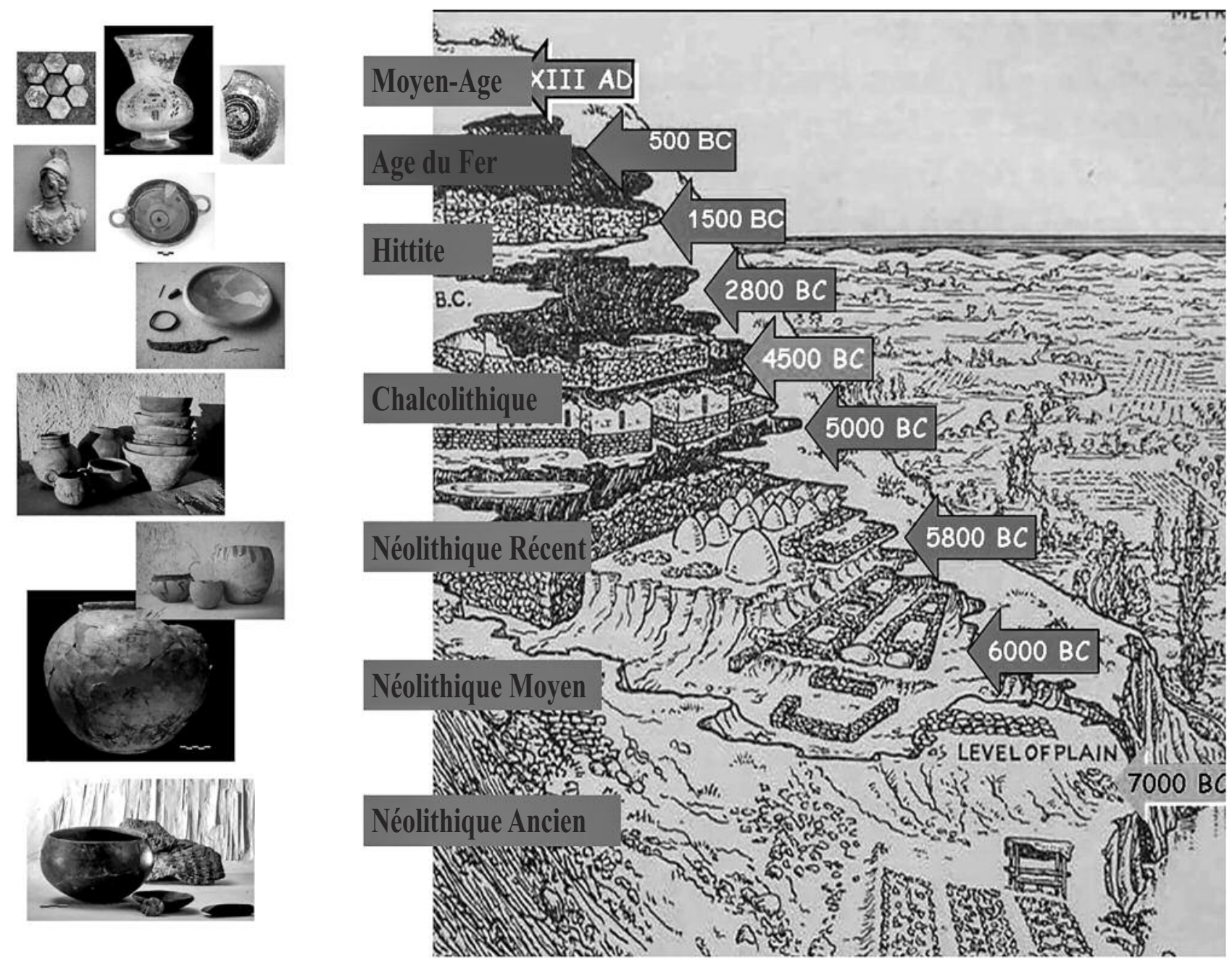

Fig. 4 : Stratigraphie et chronologie. 
ciennes habitations, celles du Néolithique Ancien (niveaux XXXIII-XXVIII), semblèrent dans ces premiers sondages être faites en hourdis, sans utilisation de pierres. Les fondations en pierres, déjà massives, de maisons aux superstructures en briques apparurent au Néolithique Moyen (XXVII-XXVI), la pierre formant toujours la base des grandes maisons absidiales du Néolithique Récent (XXV), puis des murs massifs du Néolithique final (XXIV). L'évolution architecturale, en autres éléments, permit de placer la culture néolithique de Yumuktepe dans l'orbite du développement Néolithique nord syrien ou nord levantin. Après réévaluation de la séquence stratigraphique des niveaux préhistoriques, les niveaux XXXIII à XXVIII sont désormais datés du Néolithique Ancien, XXVII et XXVI du Néolithique Moyen, XXV et XXIV du Néolithique Récent, XXIII à XX du Chalcolithique Ancien, XIX à XVI du Chalcolithique Moyen, XV à XIIB du Chalcolithique Récent et XIIA du Bronze Ancien ${ }^{19}$.

Le niveau XVI, du Chalcolithique Moyen, fut déjà l'objet d'une première réévaluation dans la première phase des nouvelles fouilles : si Garstang pensait que l'occupation de cette époque ne dépassait pas l'espace compris à l'intérieur du périmètre défini par le mur fortifié, il devenait désormais clair que l'occupation s'étendait au-delà de la citadelle et qu'une "ville" basse construite sur des terrasses se distinguait de la "ville" haute ${ }^{20}$.

L'absence d'autres niveaux Bronze Ancien que le niveau XIIA dans les fouilles anciennes, en contraste avec leur forte représentation à Tarse, avait été interprétée comme le déplacement d'un centre de gravité vers un autre, de Yumuktepe à Tarse, au début de l'âge du Bronze. Cette explication est désormais remise en cause par la mise au jour de niveaux Bronze Ancien dans les nouvelles fouilles ${ }^{21}$. L'idée que Tarse aurait joué un rôle politique et économique plus important que Mersin pendant l'âge du Bronze Ancien repose donc peut-être sur le choix et le hasard des parties fouillées des höyük $k^{22}$. Ainsi, dans un chantier septentrional de Yumuktepe, une terrasse Bronze
Ancien fut mise en évidence et datée au $\mathrm{C}_{14}$ de 2880-2470 23 .

$\mathrm{Au}$ Nord-Ouest, lors des anciennes fouilles, Garstang nomma "hittites" deux périodes bien séparées par un mur d'enceinte à caissons (la "fortification hittite") et une "plateforme" repérée à l'extérieur de ce mur, tous deux mis au jour dans le niveau VII. Il regroupa les niveaux inférieurs XI à VIII dans une même période "hittite cilicienne", qu'il data des environs de 2000 à 1500 av. J.-C. Considérant apparemment le niveau VIII comme un niveau de transition, il l'inclut aussi dans la période suivante, dite "hittite impériale", datée des environs de 1500 à 1200 av. J.-C. et qui regroupait les niveaux VIII-VII à V ${ }^{24}$. Dans le chantier sud, la synchronisation avec la première génération de fouilles fut fondée sur l'identification des restes d'un grand mur avec le reste du mur d'une hypothétique enceinte du niveau IX des anciennes fouilles, d'un remblai avec la "plateforme" attribuée par Garstang aux niveaux VII-V, et de la couche de destruction qui recouvrait le niveau $\mathrm{V}^{25}$. Des décalages importants doivent cependant être soulignés dans cette labellisation. Ainsi, le matériel céramique du niveau IX du chantier sud renvoie à un horizon culturel "hittite ancien" daté des environs de 1500 av. J.-C. (d'après des datations au $\mathrm{C}_{14}$ ), alors que cet horizon n'apparaît clairement qu'à partir du niveau VII des anciennes fouilles ${ }^{26}$. En conséquence, le remblai VII qui recouvre le "hittite ancien" du chantier sud ne peut pas correspondre à la "plateforme" de Garstang, d'autant que cette dernière était précisément recouverte par la couche de destruction du niveau $\mathrm{V}$, couche qui dans le chantier sud est postérieure au remblai ${ }^{27}$.

La grande découverte du chantier sud reste le grand mur évoqué plus haut et les quelques pièces qui lui étaient associées. Cet imposant mur d'enceinte était entièrement composé de briques crues sur une largeur de 2,50 m, sans aucune fondation en pierre mais avec deux alignements de pierres fluviales adossés à la brique, l'un à l'intérieur, l'autre, plus large et mieux agencé, à l'extérieur, qui représentait vraisemblablement un mur de soutènement ${ }^{28}$. Les

19) Caneva 2004a ; 2004b;2004c ;2010b;2010c.

20) Caneva 2004c : 57, 60-62 ; fig. 6a ; 2010c.

21) Palumbi 2010.

22) Caneva et Sevin $2004: 171$.

23) Palumbi $2010: 51$

24) Garstang 1953 : vii, 209, 237

25) Sevin et Köroğlu, K. 2004 : 74-76 ; Manuelli 2010 : 59-60.

26) Jean $2006: 325-330 ; 2010: 194-200,365-381$

27) Jean $2010: 201-202,379-381$.

28) Sevin et Köroğlu, K. 2004 : 74 ; Manuelli 2010 : 59-60 ; Jean 2010 : 195-197. Dans Manuelli 2010, p. 60, il est écrit par erreur que le mur faisait environ $8 \mathrm{~m}$ de large. L'estimation de 1,40 m pour l'épaisseur de ce mur dans Sevin et Köroğlu, K. 2004, p. 74, peut vraisemblablement s'expliquer par son repérage à une altitude supérieure à celle de sa base dévoilée plus récemment. 


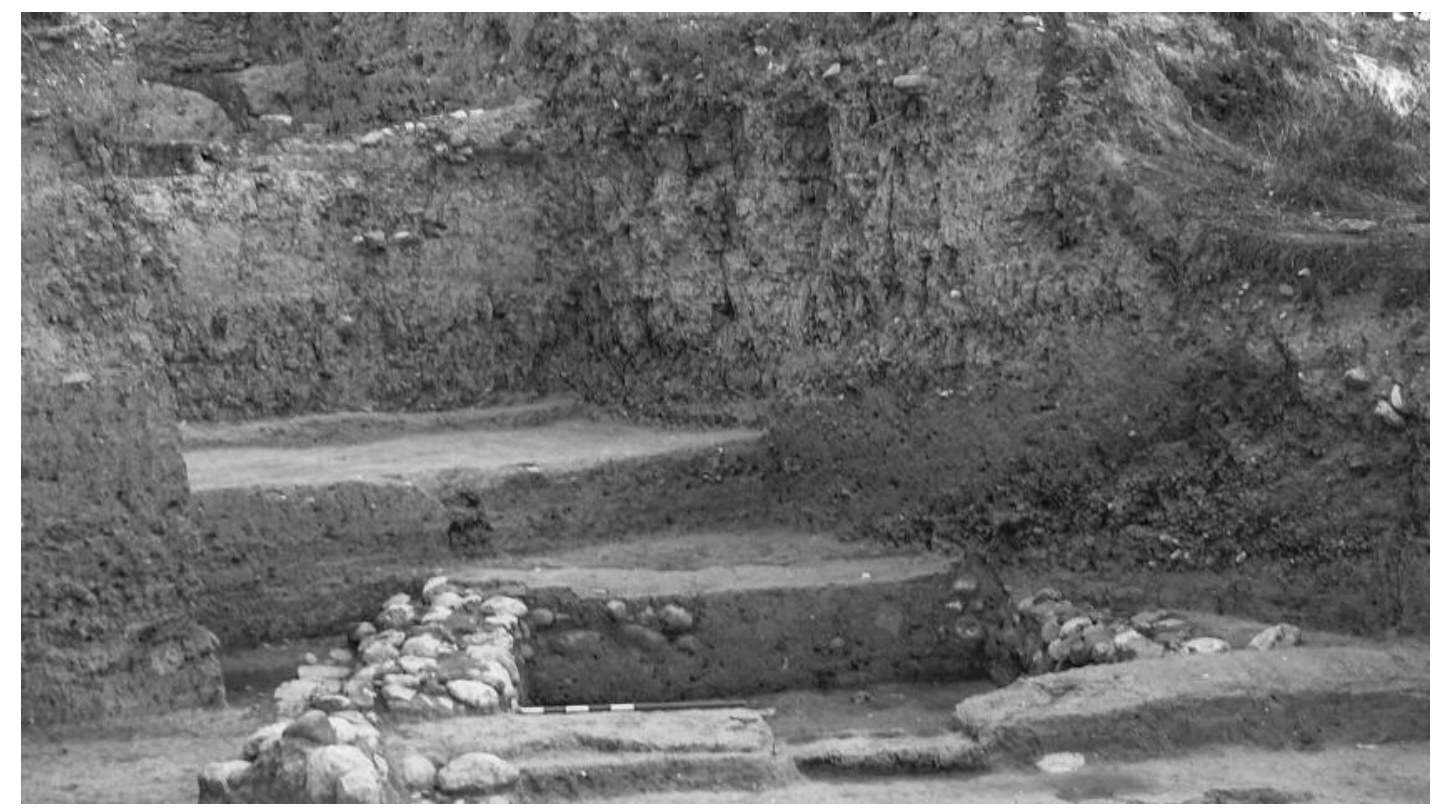

Fig. 5 : Les ruines du mur hittite : écroulement, nivellement et accumulation du remblai.

sections est et ouest du chantier montrent bien que ce mur, qui devait être très élevé, a brûlé et s'est effondré (probablement des deux côtés, mais seule la partie interne est restée : Fig. 5). Le long et à l'intérieur de cette enceinte, une rangée de deux pièces adjacentes fut dégagée, à laquelle, en parallèle, un second alignement de trois pièces était accolé au Nord. La pièce orientale de la première rangée communiquait vers le Nord par une marche avec la pièce centrale du second alignement. Consistant en armes (pointes de flèches et de lances) et en vaisselle apparemment standardisée (essentiellement des bols à lèvre rentrée, qui servirent peut-être à distribuer des rations alimentaires), le matériel exhumé dans ces différentes pièces suggère qu'un corps de gardes y était installé. Or, si ce matériel montre indiscutablement l'entrée de Yumuktepe dans l'orbite hittite, le mur d'enceinte se distingue des murs à caissons typiques des centres hittites d'Anatolie centrale. Sa composition en briques, le double alignement (partiel) de ses pièces intramuros et l'apparente absence d'architecture en-deçà de ces pièces rappelle étran- gement la configuration du mur de fortification du niveau XVI. S'agit-il de l'écho d'une tradition locale remontant au Chalcolithique Moyen? Par ailleurs, la découverte dans ce niveau "hittite ancien" du chantier sud de plusieurs fragments de Red Lustrous Wheelmade Ware importée mais aussi d'imitation locale et d'une possible variante plus grossière ${ }^{29}$, à une époque où elle n'est pas attestée plus à l'Est dans la Çukurova ${ }^{30}$, semble indiquer que Yumuktepe entretenait des liens commerciaux et culturels étroits avec ses voisins occidentaux dont, peut-être, le port d'Ura (Silifke ?) ${ }^{31}$. L'appartenance de Yumuktepe au Kizzuwatna et sa position près de la frontière occidentale de ce royaume explique en grande partie ces liens privilégiés avec l'Ouest, liens qui semblent encore renforcés au Bronze récent II, comme en atteste la présence de bols en "céramique orange" et présentant une rainure à l'extérieur de la lèvre (Fig. 6) ${ }^{32}$ et celle d'une céramique locale peinte, composée principalement de jarres à bord carré ${ }^{33}$, et dont les plus proches parallèles proviennent des niveaux du Bronze récent II de Kilise Tepe, dans la vallée du Göksu ${ }^{34}$.

29) Manuelli $2009: 258-260$, fig. 2.

30) La Red Lustrous Wheel-made Ware apparaît dès le Bronze Récent I à Kilise Tepe et, bien qu'en très faible quantité, à Yumuktepe, alors qu'elle n'apparaît qu'au Bronze Récent II dans le reste de la Cilicie plane, en dehors de Kinet Höyük où un tesson provient d'un niveau Bronze Récent I (Kozal 2015 : 55).

31) Sur l'aspect commercial, voir aussi Köroğlu, K. 2010.

32) Sevin et Köroğlu, K. $2004: 80$, fig. 6 ; 8.

33) Sevin et Köroğlu, K. 2004 : 80, fig. 7 ; 9-10.

34) Sevin et Köroğlu, K. 2004 : 80. Pour une datation de la céramique locale peinte de Yumuktepe au BR II, voir en particulier Jean $2010: 378-379$. 


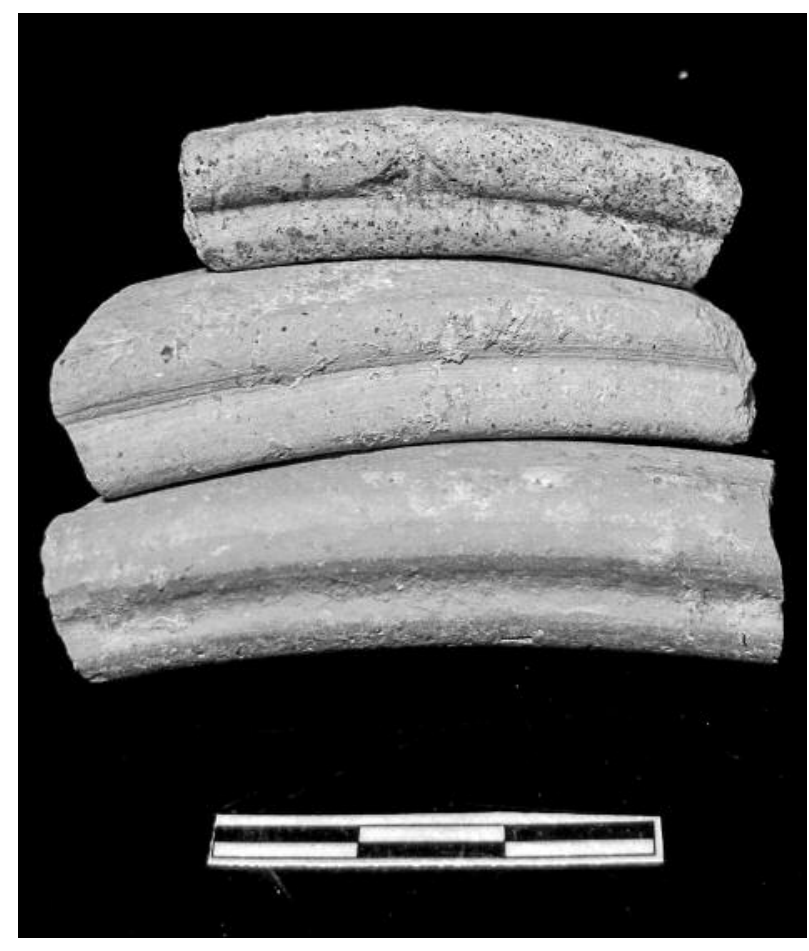

Fig. 6 : Les "bols à rainure" en "céramique orange".

Pour ce qui est du passage de l'âge du Bronze à l'âge du Fer, ni les fragments de vases retrouvés, ni la stratigraphie - incertaine - ne permettent d'aboutir à la conclusion qu'il y eut une occupation pendant le Fer Ancien, en tout cas pas immédiatement après le Bronze Récent. Garstang attribua les niveaux IV et III à l'âge du Fer ou aux "Early Greek Settlements", qu'il data de 1150 à 500 av. J.-C. ${ }^{35}$. Toutefois, selon Barnett, le matériel céramique du niveau III appartenait aux $7^{\mathrm{e}}$ et $6^{\mathrm{e}}$ siècles, et celui du niveau IV principalement au $8^{\mathrm{e}}$ siècle, une occupation effective du $11^{\mathrm{e}}$ au $9^{\text {e }}$ siècles av. J.-C. n'ayant pu être démontrée ${ }^{36}$. D'après Mee, qui réétudia les fragments considérés comme "hellado-ciliciens" par Gjerstad et submycéniens par Seton-Williams ${ }^{37}$, ceux-là ne semblaient pas être d'inspiration mycénienne et semblaient très tardifs ${ }^{38}$. Pendant la seconde génération des fouilles, des niveaux du Fer furent mis au jour sous les niveaux médiévaux dans les chantiers ouverts au Sud et sur le sommet du site mais les fondations des constructions et les tombes médiévales endomma-

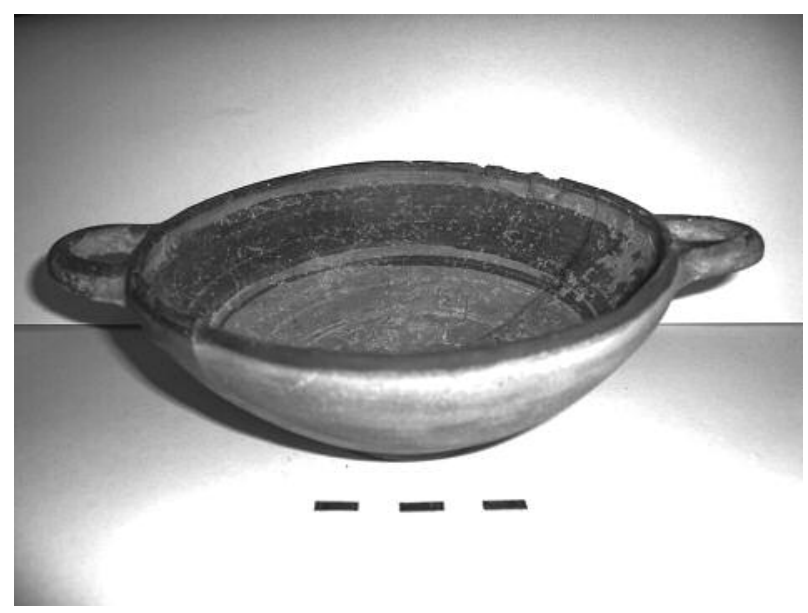

Fig. 7 : Kylix de l’âge du Fer.

gèrent tellement les niveaux inférieurs qu'une stratigraphie de l'âge du Fer n'a pu être établie. Du matériel céramique du Fer Moyen et principalement du $6^{\mathrm{e}}$ siècle av. J.-C. fut malgré tout exhumé (Fig. 7), qu'il s'agisse de fragments de vases de style géométrique, orientalisant ou chypriote, auxquels il faut ajouter des fragments de vases à figures rouges de l'Attique ${ }^{39}$.

Malgré l'absence d'architecture et le faible nombre de tessons retrouvés, Yumuktepe fut aussi occupé à l'époque hellénistique. La présence, en particulier, de fragments d'amphores semble indiquer que le site était impliqué dans les commerces du vin, d'huile d'olive et d'aliments secs, et davantage en relation avec la péninsule grecque, les îles égéennes, Chypre et la côte syrienne, qu'avec l'Anatolie centrale. Quant à l'époque romaine, le matériel trouvé au sommet du site laisse supposer qu'un petit village y était installé, l'absence de bâtiments monumentaux s'expliquant aisément par la préférence des Romains à s'installer dans des zones planes. Par la suite, il est possible que le site ait connu un abandon entre les $5^{\mathrm{e}}-6^{\mathrm{e}}$ siècles environ et le $10^{\mathrm{e}}$ siècle ap. J.-C.

Au moment de la reprise des fouilles en 1993, une occupation médiévale fut découverte au sommet du höyük, occupation qui formait un dépôt archéologique de $3 \mathrm{~m}$ d'épaisseur ${ }^{40}$. Du niveau de construction le plus récent, labellisé Ia et daté de la seconde moitié du $12^{\mathrm{e}}$ et du $13^{\mathrm{e}}$ siècle, il ne restait quasiment

35) Garstang $1953: 252$

36) Barnett $1940: 98$ n. 3, 99

37) Gjerstad 1934 : 195, fig. 18 ; Seton-Williams 1954 :134.

38) Mee $1978: 133$.

39) Sevin et Özaydın 2004 ; Özaydın 2010.

40) Köroğlu, G. 2004 ; 2010. Pour un rappel des niveaux médiévaux dégagés par Garstang et de leur réévaluation, voir Köroğlu, G. $2004: 103$. 
rien, ses vestiges ayant été presque entièrement détruits par la transformation du höyük en parc dans les années 1960. Sur la pente nord-ouest, des habitations, des jarres de stockage et les restes d'une rue pavée de galets orientée vers le sommet de la colline furent dégagés, et datés du même niveau. Les vestiges les mieux préservés étaient ceux du niveau inférieur $\mathrm{Ib}$, datés du $11^{\mathrm{e}}$ et de la première moitié du $12^{\mathrm{e}}$ siècle. Un petit village entouré par un mur d'enceinte à caissons occupait alors le sommet du höyük. A la même époque, une église au plan cruciforme et, à côté de l'église, une chapelle funéraire furent édifiées à l'intérieur de la fortification. La plus ancienne tombe fut retrouvée à l'Ouest de l'église et consistait en un sarcophage en terre cuite, à l'intérieur duquel des monnaies byzantines en bronze datant du règne commun de Basile II et de Constantin VIII (9761025 ) reposaient près du squelette. Les autres tombes mises au jour contenaient parfois deux squelettes, parfois beaucoup plus. Les squelettes étaient allongés, la tête orientée vers l'Ouest; des coupes en verre, des flacons à parfum, des bols émaillés, des assiettes, des boucles d'oreilles, des bracelets, des croix étaient déposés comme dons funéraires. Après une utilisation d'environ cent ans, l'église et les habitations furent transformées en dépôts, ateliers et cuisine. A partir de la seconde moitié du $12^{\mathrm{e}}$ siècle, une grande partie de la zone orientale du sommet du höyük ayant servi de cimetière, il est possible que l'habitat fût déplacé vers les pentes et la plaine au pied du höyük.

Malgré, d'une manière générale, une architecture peu élaborée et un agencement aléatoire des habitations, un matériel riche et varié fut retrouvé dans les maisons, qui reflétait les relations étroites établies au Moyen-Âge entre Yumuktepe et les mondes chrétiens et musulmans établis autour de l'Egée et de la Méditerranée, notamment en Syrie et en Egypte pour les derniers, grâce au commerce maritime. A côté des monnaies byzantines, furent ainsi retrouvés des monnaies islamiques appartenant aux Fatimides et aux Grands Seldjoukides, et des chandeliers en verre en forme de vase au décor peint importés de Syrie. Par ailleurs, des techniques de décor d'un groupe de vases originaires des alentours de Beyrouth furent imitées à Yumuktepe.

\section{RESUME DES TRAVAUX DES DERNIERES ANNEES (2010-2015)}

Ces dernières années, tous les niveaux n'ont pas fait l'objet de la même attention, les recherches ayant principalement porté sur les niveaux du Néolithique Ancien, du Chalcolithique et du Bronze Ancien (Plan 1).

L'une des principales différences avec les anciennes fouilles vient de l'adoption, désormais générale dans les fouilles modernes, d'une perspective multidisciplinaire, notamment pour ce qui concerne les données archéo-ethno-botaniques, archéozoologiques et anthropologiques. A Yumuktepe, première étape dans la transmission de l'économie néolithique depuis les régions d'origine vers l'Ouest du bassin de la Méditerranée, les analyses qui se réfèrent à l'agriculture et à l'élevage sont de toute évidence particulièrement significatives : dès le début de leur installation, les communautés néolithiques venant de l'Est n'utilisèrent aucune des ressources autochtones de la région, comme l'indique l'absence dans le dépôt archéologique de restes d'animaux sauvages, donc de chasse et de pêche, leur économie reposant entièrement sur l'agriculture et l'élevage. Une analyse des espèces de blé cultivées à Yumuktepe a mis récemment en lumière des caractéristiques qui permettent de mieux suivre les trajectoires de transmission des techniques agricoles et, donc, d'insérer les relations culturelles suggérées par le matériel archéologique dans de possibles réseaux d'interaction économique de ces premières communautés ${ }^{41}$.

Les recherches les plus récentes dans les niveaux néolithiques montrent que l'absence de véritables structures dans les premières phases n'était qu'apparente. L'élargissement des sondages dans la zone nord-est (carrés E3/F3), immédiatement au Nord du sondage "A" de Garstang, révéla finalement un ensemble villageois cohérent : d'abord, la présence d'une grande zone de stockage, probablement une cour, présentant une concentration de silos, dont ne furent retrouvées que les bases circulaires pavées de galets, mais qui devaient être construits en hauteur selon une forme cylindrique, avec des parois en terre appliquée à une structure en branches tressées, comme suggéré par les exemples ethnographiques connus dans le Sud-Est anatolien (Burhan Ulaş, comm. pers.) ; ensuite, à la limite septentrionale de cette cour, des murs de fondations en pierres surmontées d'une superstructure en briques crues, dont c'est la première apparition à Yumuktepe dans ces niveaux et qui attestent l'existence de maisons solides, probablement à plan rectangulaire, à côté des cabanes à plan circulaire (Fig. 8).

Dans la phase suivante du Néolithique Ancien, malheureusement très perturbée par des sépultures 


\section{Mersin-Yumuktepe 2015}

\begin{tabular}{|l|l|l|l|l|l|l|l|l|l|l|l|l|l|l|l|l|l|l|l|l|l|l|l|l|l|l|l|l|l|}
\hline & 1 & 2 & 3 & 4 & 5 & 6 & 7 & 8 & 9 & 10 & 11 & 12 & 13 & 14 & 15 & 16 & 17 & 18 & 19 & 20 & 21 & 22 & 23 & 24 & 25 & 26 & 27 & 28 & 29 \\
\hline
\end{tabular}

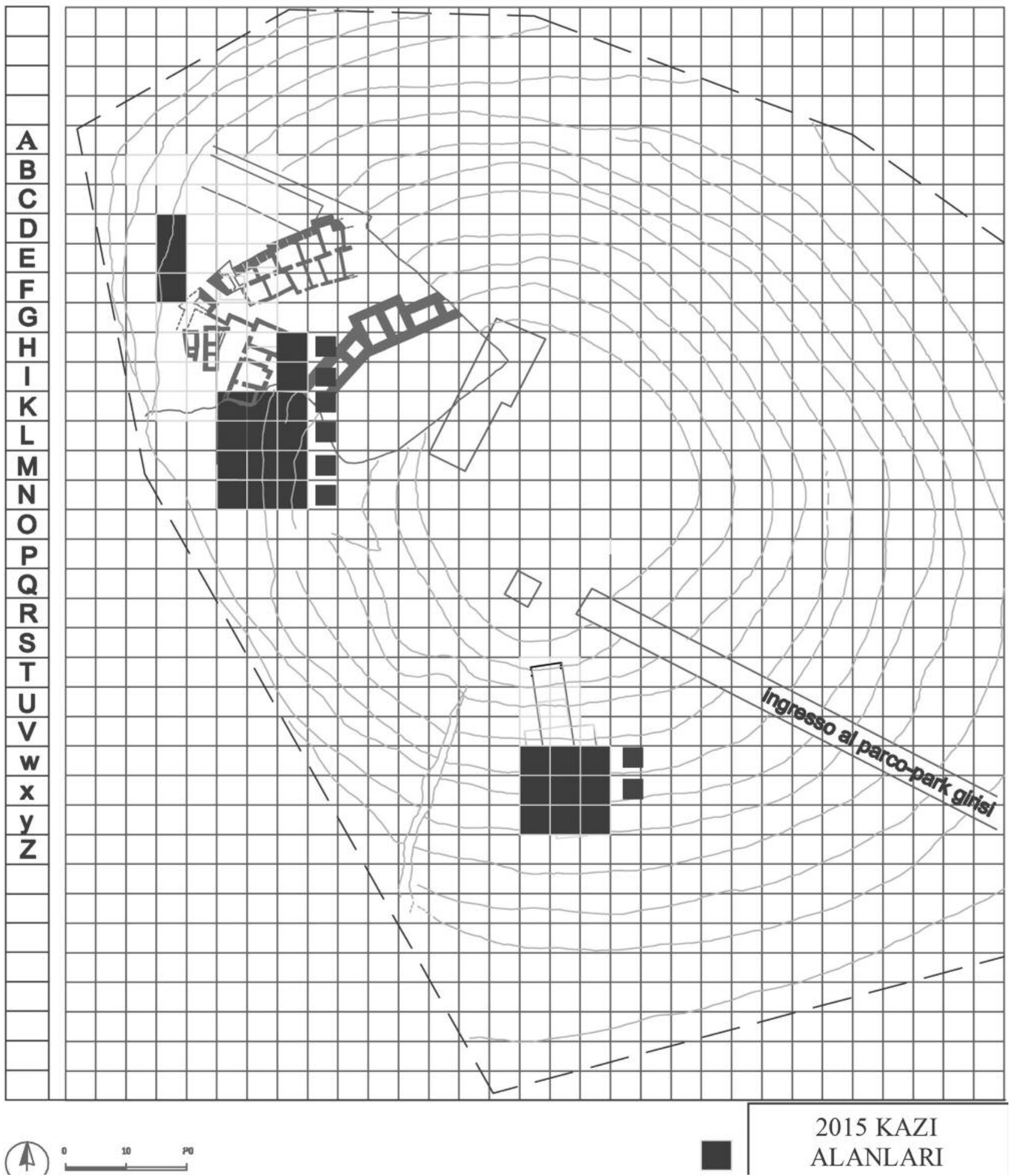

Plan 1 : Yumuktepe. Plan général indiquant les carrés fouillés jusqu'en 2015. 


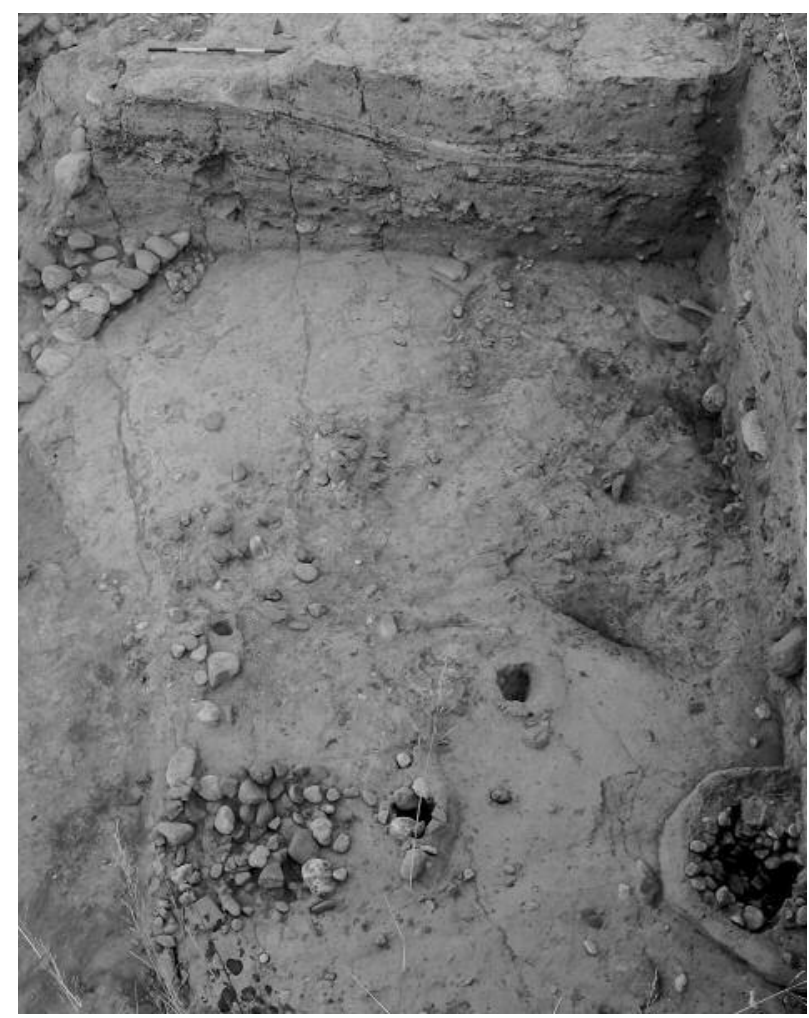

Fig. 8 : Néolithique Ancien : les silos et le mur à fondations en pierres et superstructure en briques (haut gauche). et des terrasses de différentes époques, les restes d'un sol de maison sur lequel se trouvait un foyer rectangulaire, au fond pavé de galets, furent récemment dégagés. Le sol et le foyer étaient recouverts d'un enduit peint en rouge, plusieurs fois renouvelé, ce qui renvoie ainsi aux sols rouges typiques du Néolithique acéramique de l'Anatolie centrale et du Proche-Orient (Fig. 9). Un espace adjacent et peutêtre fonctionnellement lié à cette maison montrait une concentration inusuelle de lames, d'éclats et de déchets de taille de silex et d'obsidienne : l'homogénéité de la matière première et la séquence de taille simple et remontable dans quelques cas suggèrent que cet espace était un atelier de taille.

La céramique de ces phases montre des formes typiquement globulaires, rarement ouvertes, avec des parois très fines, à bord simple, montées au colombin à partir d'une pâte fine, gritty, marron foncé, bien brunie et bien cuite. A côté de nombreux instruments en os, furent trouvés des sceaux aux motifs linéaires simples et des pintadere en os et en pierre.

Les phases suivantes du Néolithique n'ont pas fait l'objet de fouilles pendant les dernières campagnes. En revanche, une construction de la fin de la séquence néolithique, datée au $\mathrm{C}_{14}$ de 5500 av. J.-C. et caractérisée par une céramique Halaf, fut mise au jour dans un petit secteur de la zone nord-ouest, juste audessous du niveau XVI. Elle était composée d'une

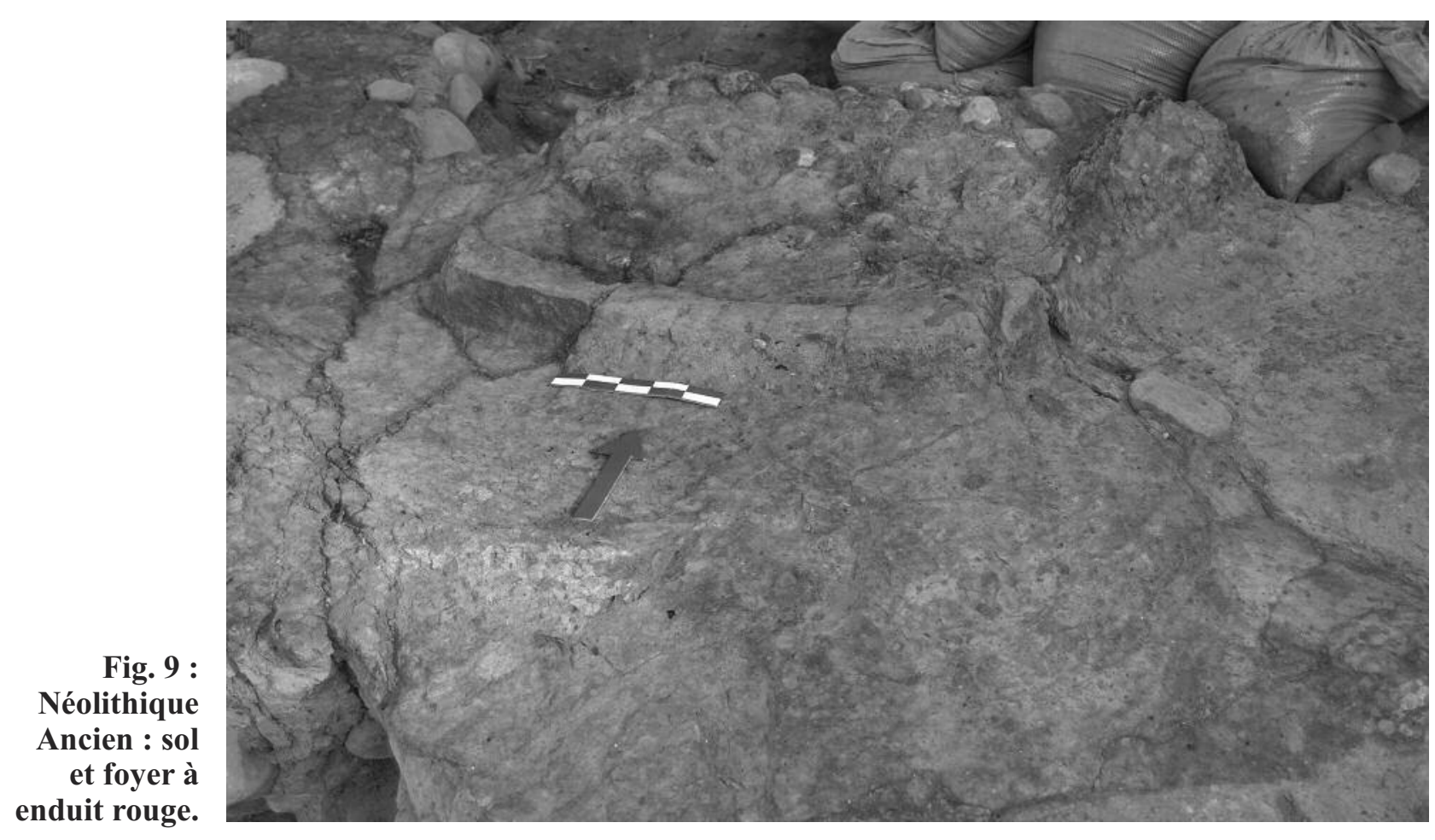




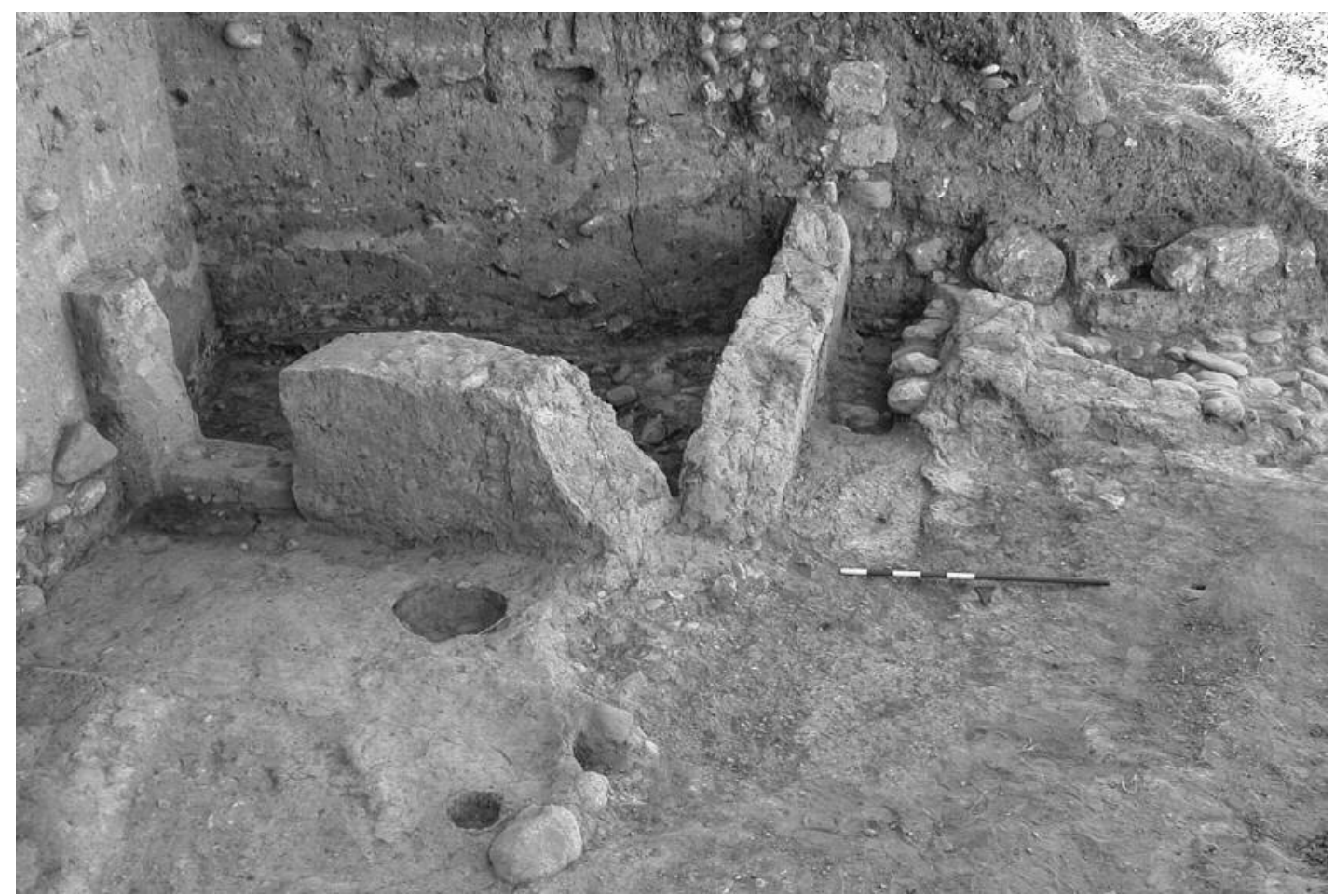

Fig. 10 : Maison Halaf coupée par une terrasse médiévale (blocs taillés).

petite pièce rectangulaire et d'une cour partiellement pavée de galets plats, dans laquelle se trouvait un foyer en forme de fer à cheval. Comme dans la description de ces niveaux dans les anciennes fouilles (niveaux XXIII-XXII) ${ }^{42}$, les murs étaient fins et consistaient en des fondations en pierres fluviales surmontées par une seule rangée de briques caractérisées par leur couleur jaune (Fig. 10). Dans les dernières campagnes, les tracés de ces murs avaient souvent été rendus visibles par des terrasses ou des fosses postérieures qui les avaient rasés, sans qu'un plan cohérent de cet habitat ne soit, pour l'instant, reconstituable.

Les résultats majeurs des dernières campagnes concernent les différentes phases du Chalcolithique (Plan 2). Les fouilles dans le niveau XVI furent reprises à partir de la campagne 2012, et mirent en lumière la continuité structurale de la citadelle dans toute la zone au Sud de la porte d'accès et du grand bâtiment mis au jour pendant la première génération de fouilles. Le même système architectural, avec un large mur en briques qui forme un arc à contour segmenté et à l'intérieur et contre lequel s'appuie une série de pièces entassées, fut mis en évidence. Six nouvelles pièces, plus petites que les anciennes et pas aussi bien préservées, donnaient également sur des cours vers l'intérieur de la citadelle (Fig. 11). A la différence de ce qui était visible dans les anciennes fouilles, il y avait très peu de matériel dans les nouvelles pièces, même dans celles qui avaient été scellées par l'écroulement des murs au moment de la destruction du niveau. Ces pièces montrent, en outre, que plusieurs modifications avaient été apportées, en ajoutant des cloisons, en élevant le sol et, dans certains cas, en oblitérant complétement la pièce pour la reconstruire au-dessus dans le même espace : il y a donc certainement une histoire beaucoup plus dynamique de ces structures, par rapport à celle mise au jour dans les anciennes fouilles. La succession de modifications amène finalement à distinguer une véritable nouvelle phase d'utilisation, dans quelques cas directement superposée à la première, où la présence de nombreux grands fours montre peut-être la perte de la fonction défensive de la citadelle.

Les recherches concernant la caractérisation et la fonction de la citadelle furent étendues au chantier sud, de façon à vérifier si la fortification s'étendait sur tout le périmètre de la colline et si des maisons en terrasse avaient aussi été établies sur le versant sud. Au-dessous de l'installation du grand mur "hittite ancien" en briques, et de ce qui reste de

42) Garstang $1953:$ :71-73. 


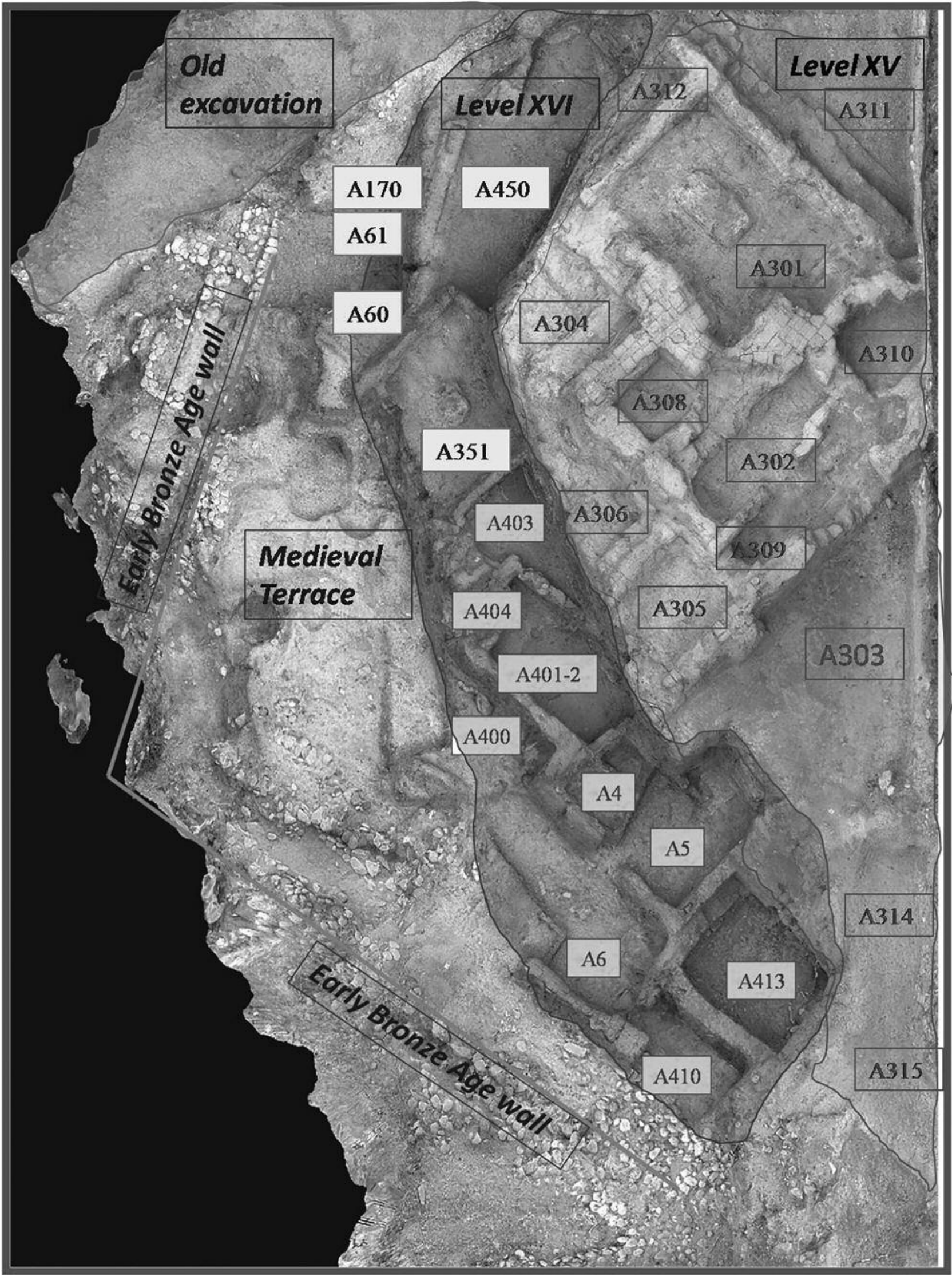

Plan 2 : Yumuktepe. Photo-plan des niveaux XV et XVI. 


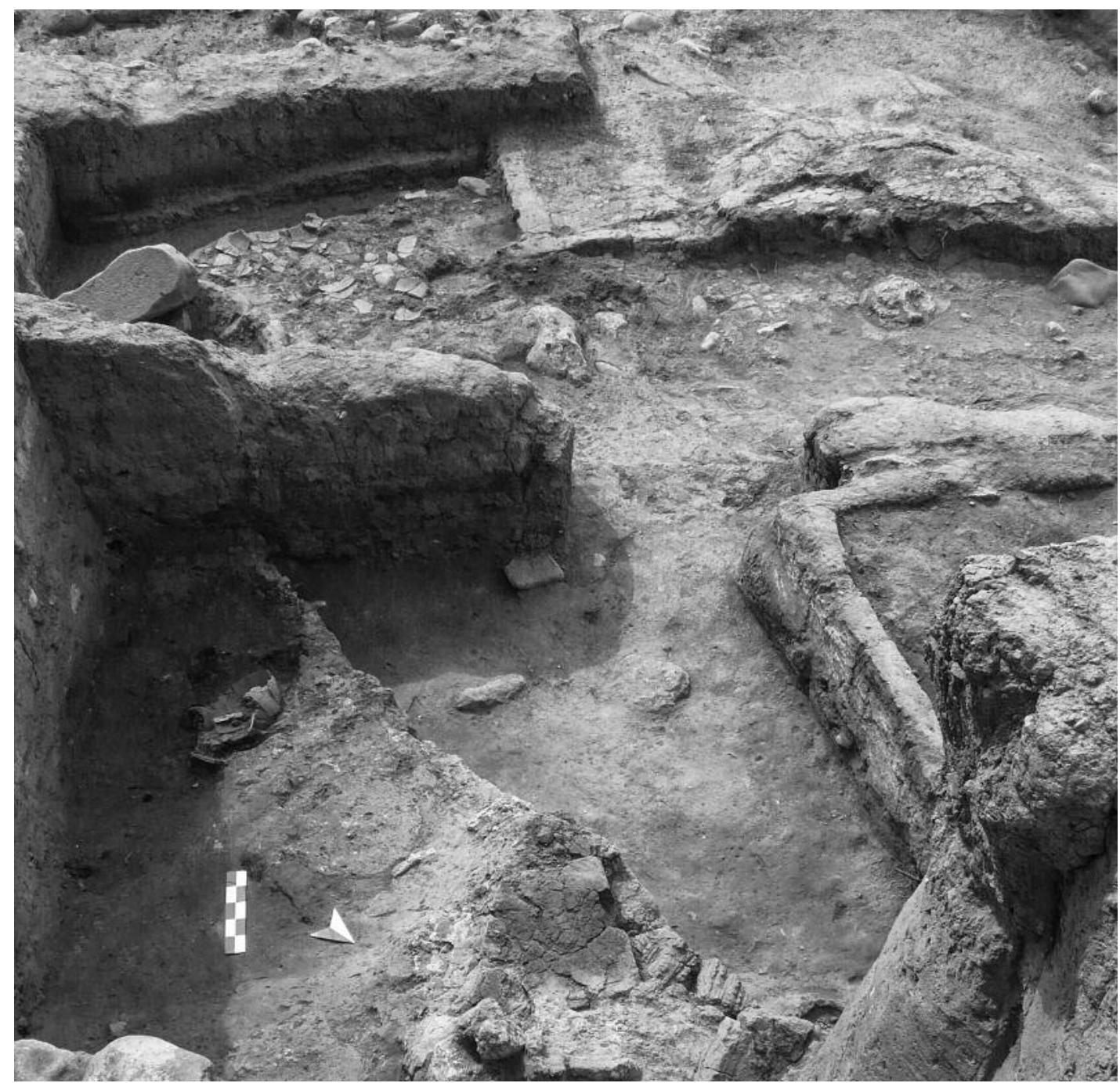

Fig. 11 : Deux pièces de la citadelle.

quelques petites maisons du niveau $\mathrm{XV}$, en partie détruites et en partie englobées par le mur même, un tas de briques brûlées a révélé la présence d'un important niveau d'incendie. La fouille de ce niveau a ensuite confirmé qu'il s'agissait des structures du niveau XVI, avec, là encore, un robuste mur externe en briques, reposant sur des fondations en grandes pierres calcaires (Fig. 12). A l'intérieur du mur, deux petites pièces communicantes furent dégagées (Fig. 13). La base des pierres calcaires qui longe les pièces à l'extérieur forme un angle droit où les assises montent jusqu'à former un bastion d'un mètre et demi de hauteur, qui interrompt la continuité de la fortification. Plusieurs éléments de similarité avec les anciennes fouilles suggèrent la présence ici d'une deuxième porte d'accès à la citadelle, laquelle devait donc suivre le plan circulaire à plusieurs accès typique du modèle des fortifications anato- liennes, comme encore attesté plus tard dans, par exemple, le site de Demircihüyük.

Dans le chantier nord-ouest, aussi bien que dans le chantier sud, le niveau de la citadelle, notamment dans sa partie externe, fut coupé à plusieurs reprises par des murs en terrasse appartenant à différentes périodes, la première coupe provenant déjà de la phase suivante, celle du niveau XV des anciennes fouilles. Dans le chantier nord-ouest, le mur extérieur du niveau XVI fut presque entièrement enlevé pour faire place à un grand mur de soutènement en pierres de rivière, qui s'appuyait obliquement sur la pente. $\mathrm{Ce}$ mur et les ruines qu'il contenait formèrent la base d'une grande plateforme en briques crues, d'une hauteur d'environ un mètre et demi, construite par-dessus. Cette plateforme consistait en plusieurs assises de briques avec des poutres en bois intercalées et quelques rangées de pierres de calage insérées 

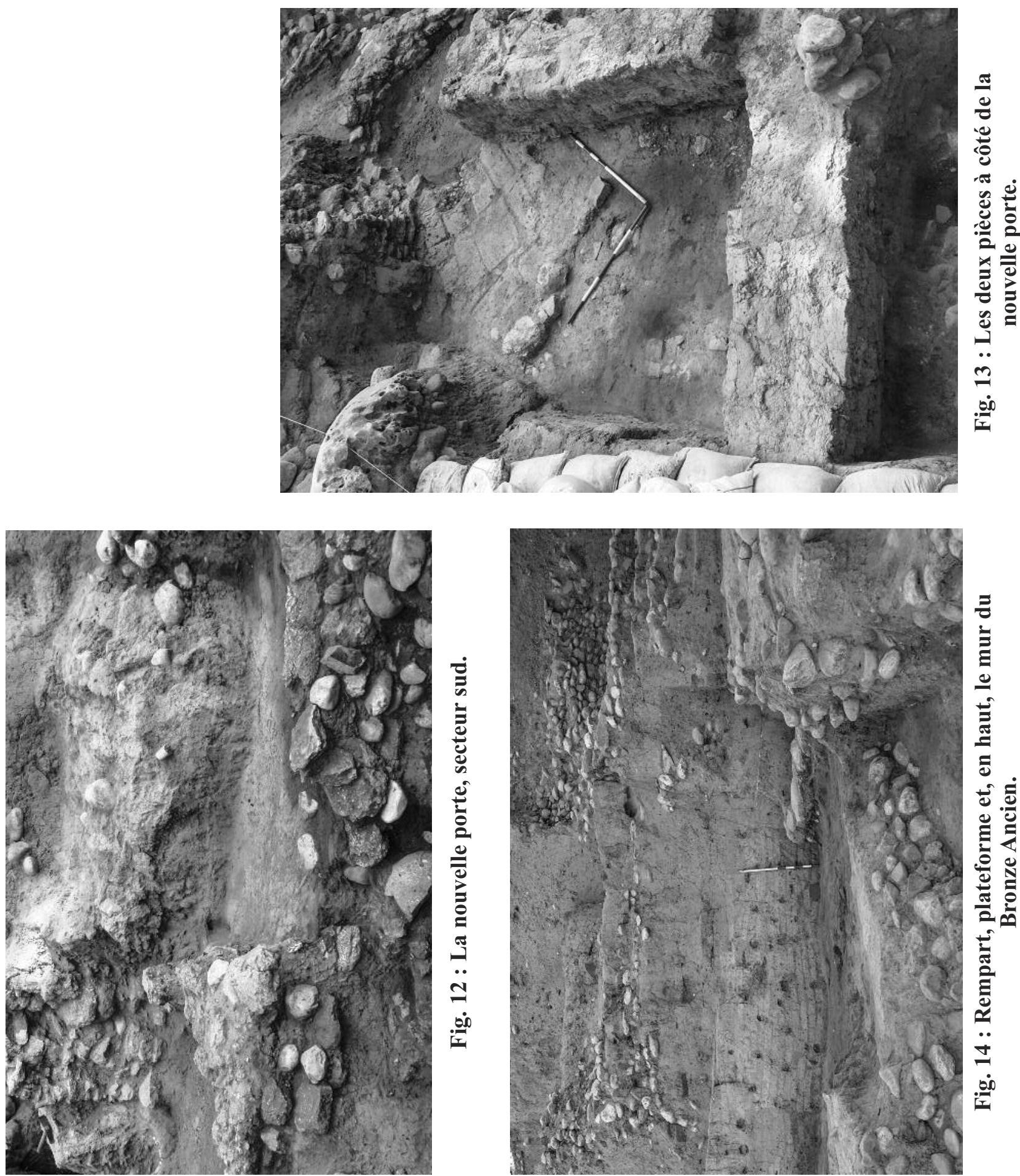


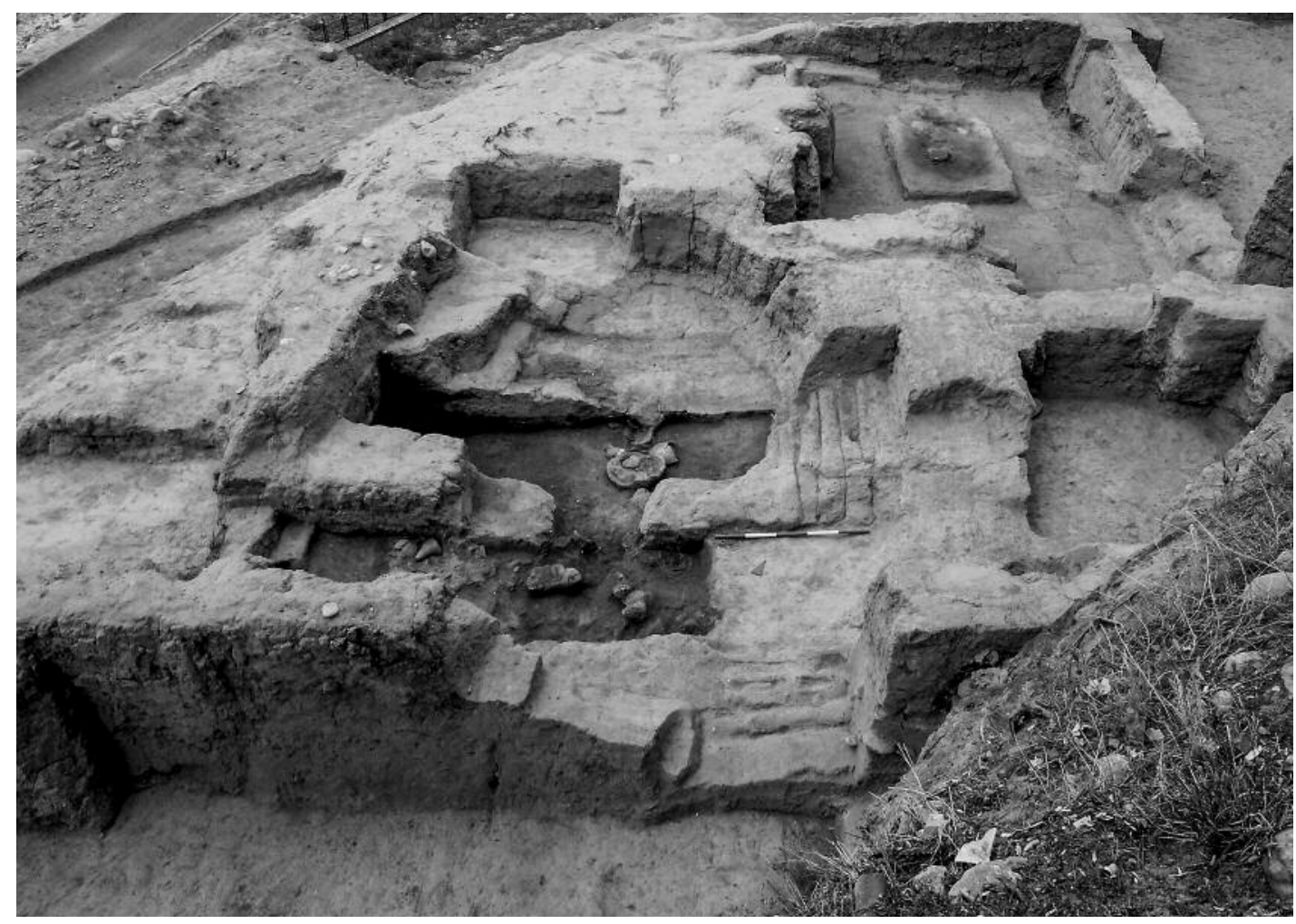

Fig. 15 : Le "palais" du Chalcolithique Récent.

pour soutenir le poids de la construction sur le côté externe, vers la pente (Fig. 14). La fonction de cette plateforme était apparemment de niveler les ruines irrégulières de la destruction de la citadelle, et de former un socle robuste et une surface plane servant à recevoir un grand bâtiment. La découverte du fait que cette surface était beaucoup plus large que le plan du bâtiment, et que la rue qui passait à l'extérieur de ce dernier était également construite par-dessus le socle de briques, permet d'avancer l'hypothèse que la plateforme s'étendait à toute la surface de la colline. Cette découverte récente permet maintenant de mieux interpréter la description de l'architecture du niveau XV dans le rapport des anciennes fouilles, où il est fait référence à une tour massive en briques d'environ 15 mètres de large ${ }^{43}$. Il s'agit très vraisemblablement de l'extension de la même plateforme, qui d'ailleurs a été reconnue dans la section des fouilles anciennes récemment repérée sur le terrain, et qui donc pourrait avoir eu une extension totale d'au moins $2000 \mathrm{~m}^{2}$. A raison d'environ six briques par $\mathrm{m}^{2}$, le nombre de briques nécessaires pour couvrir cette surface d'une épaisseur moyenne de 1,50 m s'élève à plusieurs milliers : plusieurs milliers de briques furent ainsi préparées et mises en place avant de construire le bâtiment, pour lequel plusieurs autres milliers auront été nécessaires. Combien d'ouvriers ont dû travailler, et pendant combien de temps, pour compléter cette construction ? Qui pouvait demander un si énorme investissement de travail ?

Une réponse à ces questions est en partie contenue dans les caractéristiques techniques et stylistiques tant du bâtiment même que des objets qui étaient conservés à l'intérieur. En dehors de la technique de construction complexe de l'énorme base de l'édifice, c'est aussi la dimension même de ce dernier qui est frappante par rapport aux dimensions habituelles des maisons préhistoriques de Yumuktepe. Ensuite, comme déjà pour le plan de l'édifice central de la citadelle des anciennes fouilles d'après la lecture qu'en fit C. Breniquet ${ }^{44}$, celui de cet édifice est tripartite, selon une tradition typique des cultures obéidiennes de Syrie et Mésopotamie : il s'agit donc d'une innovation étrangère à l'Anatolie (Fig. 15). Les caractéristiques techniques de la construction à l'intérieur - pilastres/contreforts, angles à redans, niches, pavement en briques - et à l'extérieur - revêtement de blocs de calcaire carrés - sont autant 


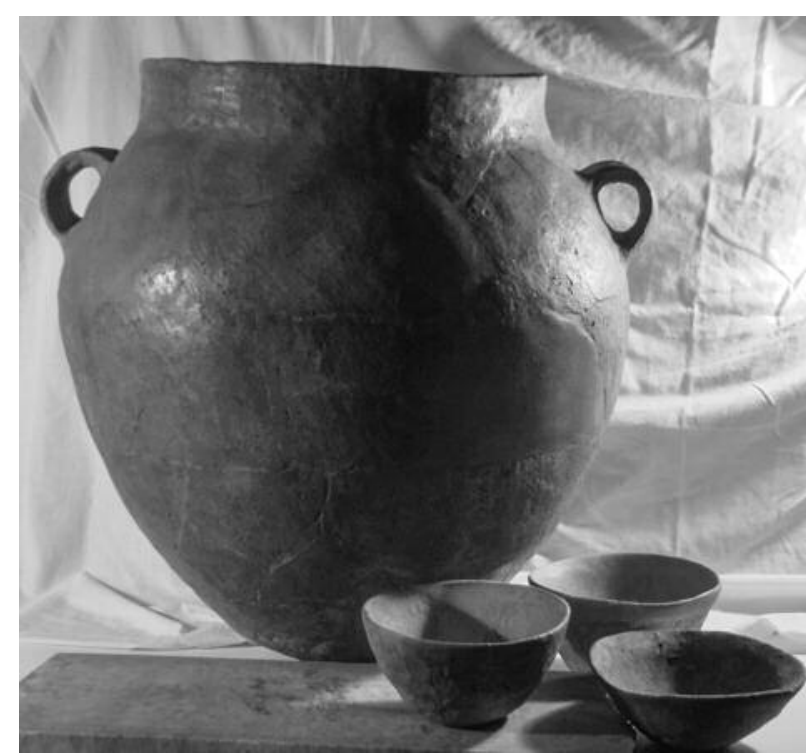

Fig. 16 : La vaisselle pour la distribution de nourriture.

d'éléments tout à fait exceptionnels, qui renvoient eux aussi à l'architecture des grandes maisons obéidiennes. L'organisation de l'espace interne répond à des fonctions spécifiques, ce qui est mis en évidence par la présence ou non de foyers dans les pièces, et par la distribution des poteries, concentrées en milliers de fragments dans les pièces latérales, mais absentes dans la salle centrale. D'autres éléments importants sont représentés par un vase de provenance vraisemblablement iranienne, une empreinte de seau, et les vaisselles produites en série : tous ces éléments sont d'habitude liés à des contextes proto-urbains, notamment au commerce de longue distance, au contrôle des mouvements des marchandises et à la production de masse. Il s'agit donc d'un bâtiment spécial, où avaient probablement lieu des cérémonies et des distributions de rations de nourriture (Fig. 16), dans un contexte visiblement lié à un modèle tout à fait préliminaire d'une élite dominante ${ }^{45}$.

La possible correspondance entre la plateforme en briques et les niveaux XV-XIV des anciennes fouilles laisse suggérer que le grand bâtiment construit par-dessus, d'abord attribué au niveau XV des anciennes fouilles, pourrait correspondre au niveau suivant, le XIII, un niveau d'ailleurs peu différencié des autres dans les anciens rapports de fouille.

Les phases qui suivent - celles de l'ancien niveau XIIB - sont très mal documentées, dans les nouvelles aussi bien que dans les anciennes fouilles, à cause d'un grand nombre de fosses de stockage

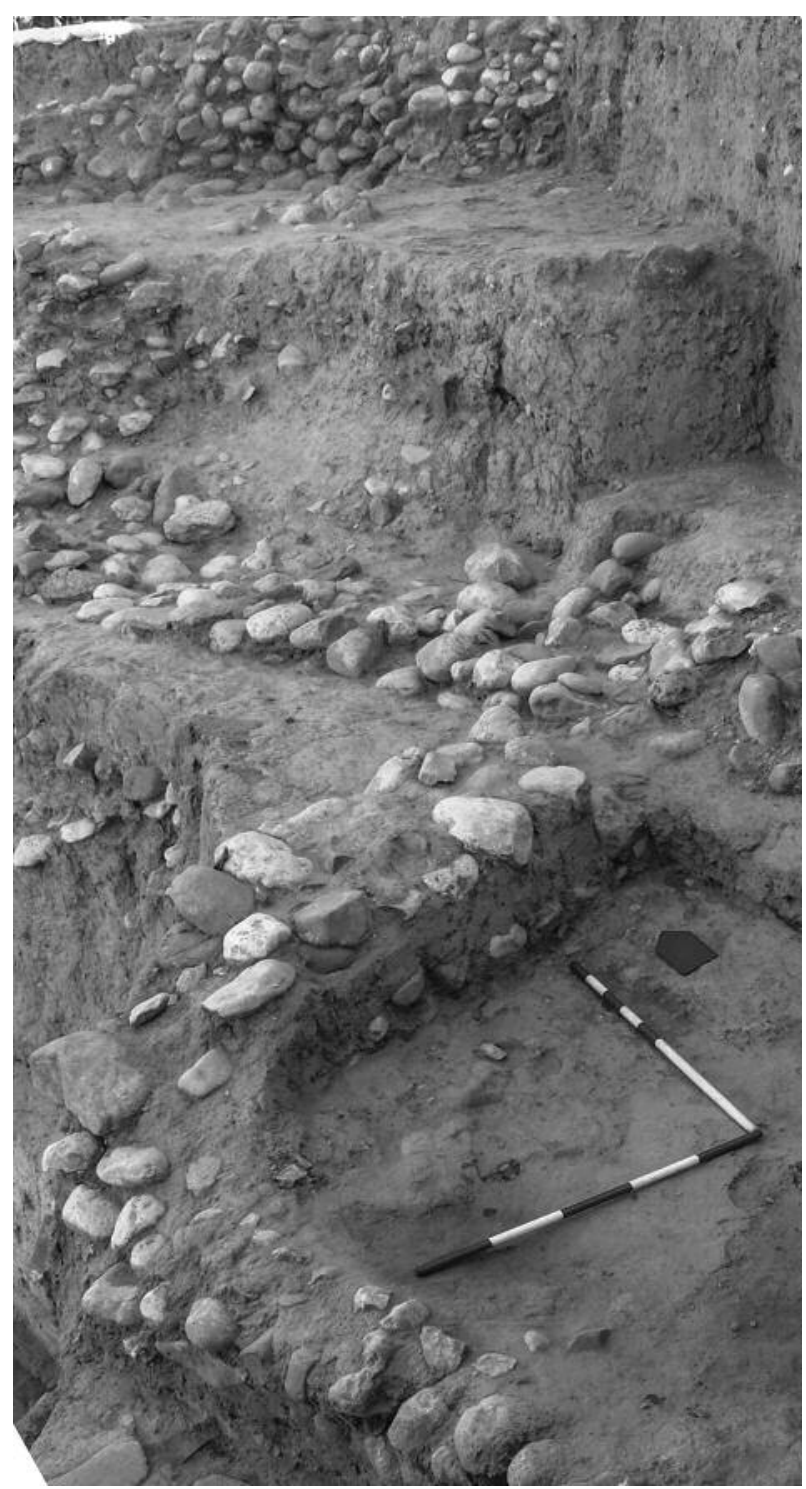

Fig. 17 : Le mur du Bronze Ancien.

successives et de l'emplacement d'un grand mur en pierres de rivière qui descend en escalier tout le long du versant nord-ouest (Fig. 17) : avec des marches profondément creusées dans la pente, ce mur coupe tous les niveaux de la séquence chalcolithique, pour ne s'arrêter que bien au-dessous de l'habitat halafien. Juste au-dessous d'un des degrés de ce mur, cependant, une petite installation a pu être conservée, creusée à son tour dans les ruines du grand bâtiment chalcolithique. Elle doit donc se situer dans le temps qui suit la destruction du grand bâtiment mais bien avant le début des nouvelles grandes installations de l'âge du Bronze. Malgré la

45) Caneva et al. 2012. 


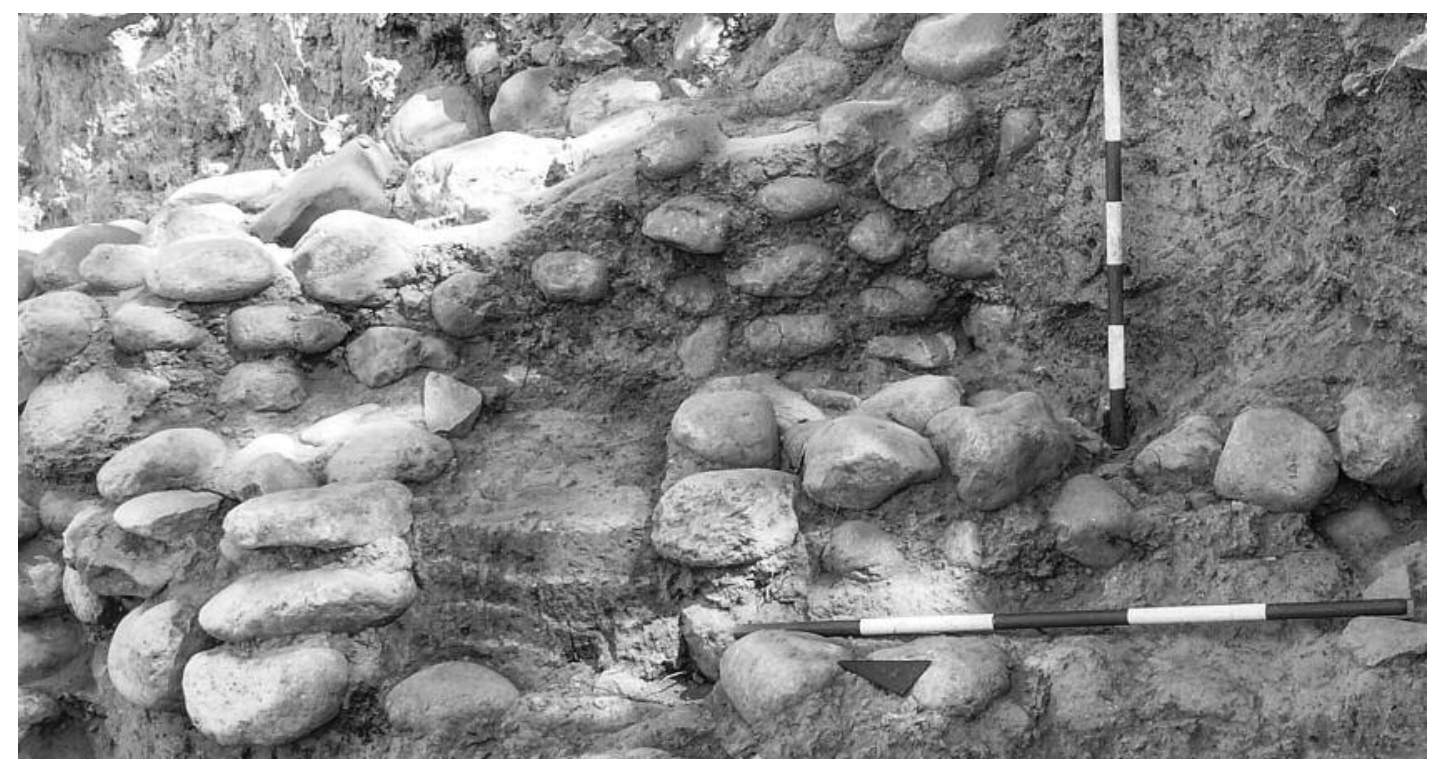

Fig. 18 : Insertion de briques crues dans le mur en pierre du Bronze Ancien.

petite surface où les traces de cette installation ont pu être repérées, il est clair qu'elle revêt des caractéristiques très marquées qui permettent de la considérer comme une occupation temporaire, probablement liée à des pasteurs semi-nomades, qui habitaient des cabanes et des abris dont il ne reste que les trous des poteaux. A côté d'un amas de bois de cerf, il y avait de grands vases en céramique grossière et une passoire pour le traitement du lait. En attendant la date $\mathrm{C}_{14}$, il est possible de présumer que cette occupation concerne les premiers siècles du quatrième millénaire, pour lequel aucune autre trace de structures n'a été repérée, ce qui suggère une longue période d'abandon du site. L'absence d'habitats au quatrième millénaire pourrait être attribuée à un vide politique qui aurait suivi la chute du système de proto-centralisation expérimentée avec le grand bâtiment, lequel était probablement anachronique et trop faible pour pouvoir se consolider et évoluer. De fait, toute l'époque d'Uruk est absente à Yumuktepe et la première véritable réoccupation du site est datée du début du troisième millénaire.

Pour ce qui concerne cette reprise d'occupation, le grand mur de fortification en pierres a déjà été mentionné. Ce mur devait constituer un revêtement de la partie haute de la colline, incluant en son sein tous les niveaux chalcolithiques. La structure devait être à casemates mais construite avec une technique très particulière, dans laquelle les rangées de pierres fluviales étaient intercalées avec des couches de briques (Fig. 18). Pendant la dernière campagne de fouilles, la partie supérieure de cette fortification a pu être reliée à un niveau d'habitat clairement attribuable, d'après la céramique, au Bronze Ancien I / II. La fouille permit la mise au jour de petites maisons rectangulaires, aux murs en briques et fondations en pierres (Fig. 19). La reconstruction du plan de la fortification et de son habitat permet maintenant de mieux interpréter les restes contemporains déjà dégagés dans la partie nord du chantier, à une élévation nettement inférieure. Il s'agit sans doute d'installations en terrasses externes à l'habitat fortifié, liées à des activités rurales, comme le montreraient les nombreuses fosses de stockage et les trous de poteaux qui suggèrent un espace de travail en plein air. Ce niveau avait été daté au $\mathrm{C}_{14}$ de 2800 av. J.-C., date confirmée par la poterie de deux contextes, qui inclue des types caractéristiques de cette époque, comme la Red Gritty Painted Ware, déjà identifiée à Tarse dans les niveaux correspondants $^{46}$ : il s'agit d'une céramique à parois extrêmement fines, excessivement cuite et au son métallique, dont la forme la plus courante est la cruche à bec verseur décorée de motifs peints en blanc, rouge et souvent polychrome, sans répertoire géométrique (Fig. 20).

Bien que cette fortification soit la plus massive du tepe, se déroulant sur une hauteur d'environ 10 mètres, elle n'a pas été reconnue dans le passé. C'est probablement sa construction en terrasses, 


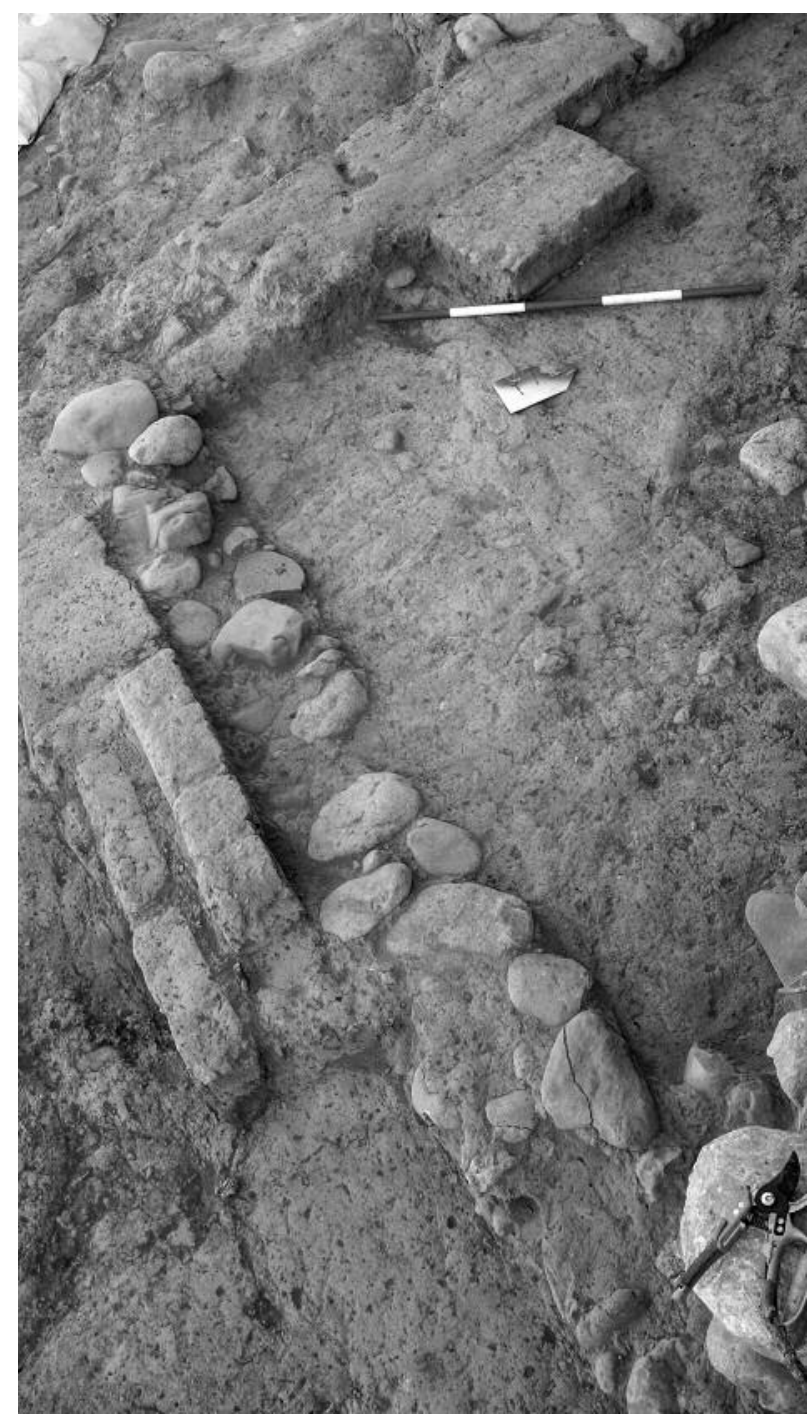

Fig. 19 : Habitat du Bronze Ancien.

dont les degrés correspondent à plusieurs niveaux, qui a empêché de la voir dans son ensemble et dans sa continuité verticale.

Ce mur fut à son tour mal conservé en raison de plusieurs coupures et obturations dues à l'aménagement d'espaces en terrasses à l'époque médiévale. Les terrasses du Moyen-Âge représentaient l'extension extramuros de l'habitat du sommet, et consistaient en installations liées à la production agricole. Renforcés en amont par des murs de soutènement en blocs de calcaire taillés, des espaces ouverts, parfois très larges, se situaient à des niveaux différents sur tout le versant de la colline, jusqu'à sa base. Aucune véritable maison n'a été reconnue et la grande

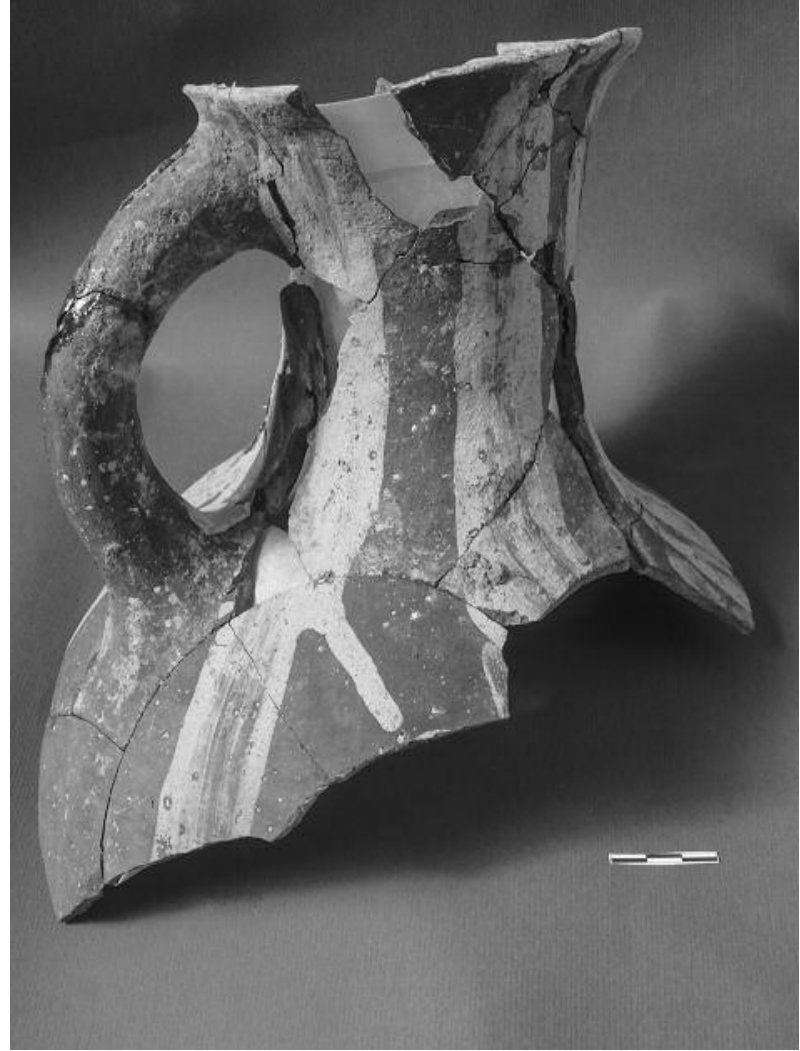

Fig. 20 : Vaisselle à peinture blanche du Bronze Ancien.

quantité de charbon de bois qui recouvrait toutes ces surfaces suggère que des poteaux avaient été utilisés pour des toitures ou des abris afin de protéger des zones de travail, où des foyers et de grands pithos enterrés dans le sol furent retrouvés ${ }^{47}$. Le matériel associé contient de la céramique peinte émaillée, datée des $12^{\mathrm{e}}-13^{\mathrm{e}}$ siècles et des objets, en fer surtout, armes, clous et outils, qui se distinguent de la richesse des dépôts dans le village du sommet (Fig. 21-22).

\section{CONCLUSION}

Comme toujours, les questions ouvertes par les nouvelles fouilles sont aussi nombreuses que les réponses apportées aux problèmes initiaux. Ainsi, si une vision beaucoup plus cohérente des habitats de toutes les phases, aussi bien que de l'économie, de la technologie et, surtout, de la chronologie, fut acquise, la topographie de ces habitats n'est encore que très partiellement reconstruite. Où sont, par

47) Un des foyers et, à proximité, le fond d'un pithos étaient attribués au niveau Halaf dans le rapport des anciennes fouilles, du fait de leur position stratigraphique (Garstang 1953 : 105). 


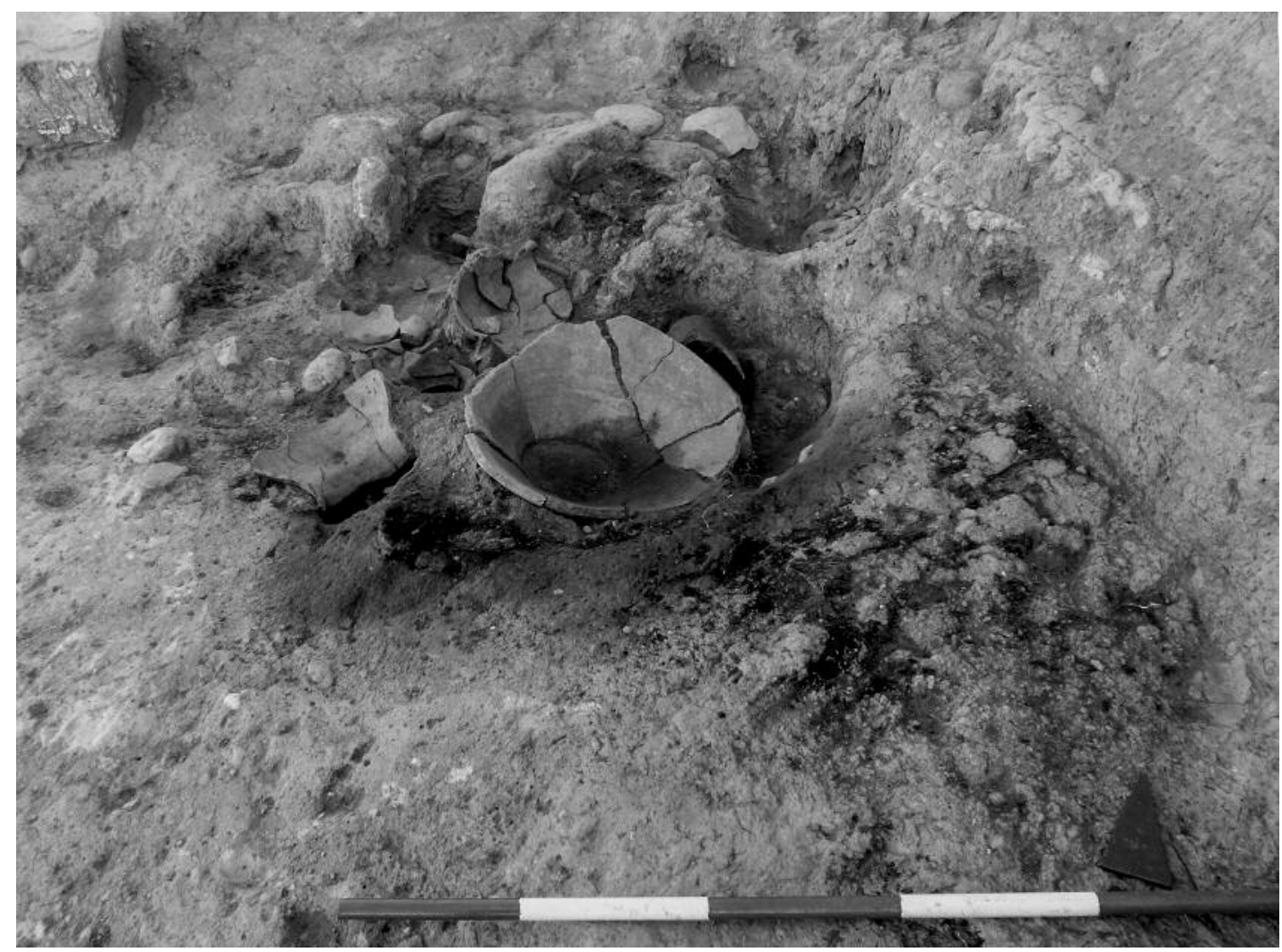

Fig. 21 : Foyer médiéval.

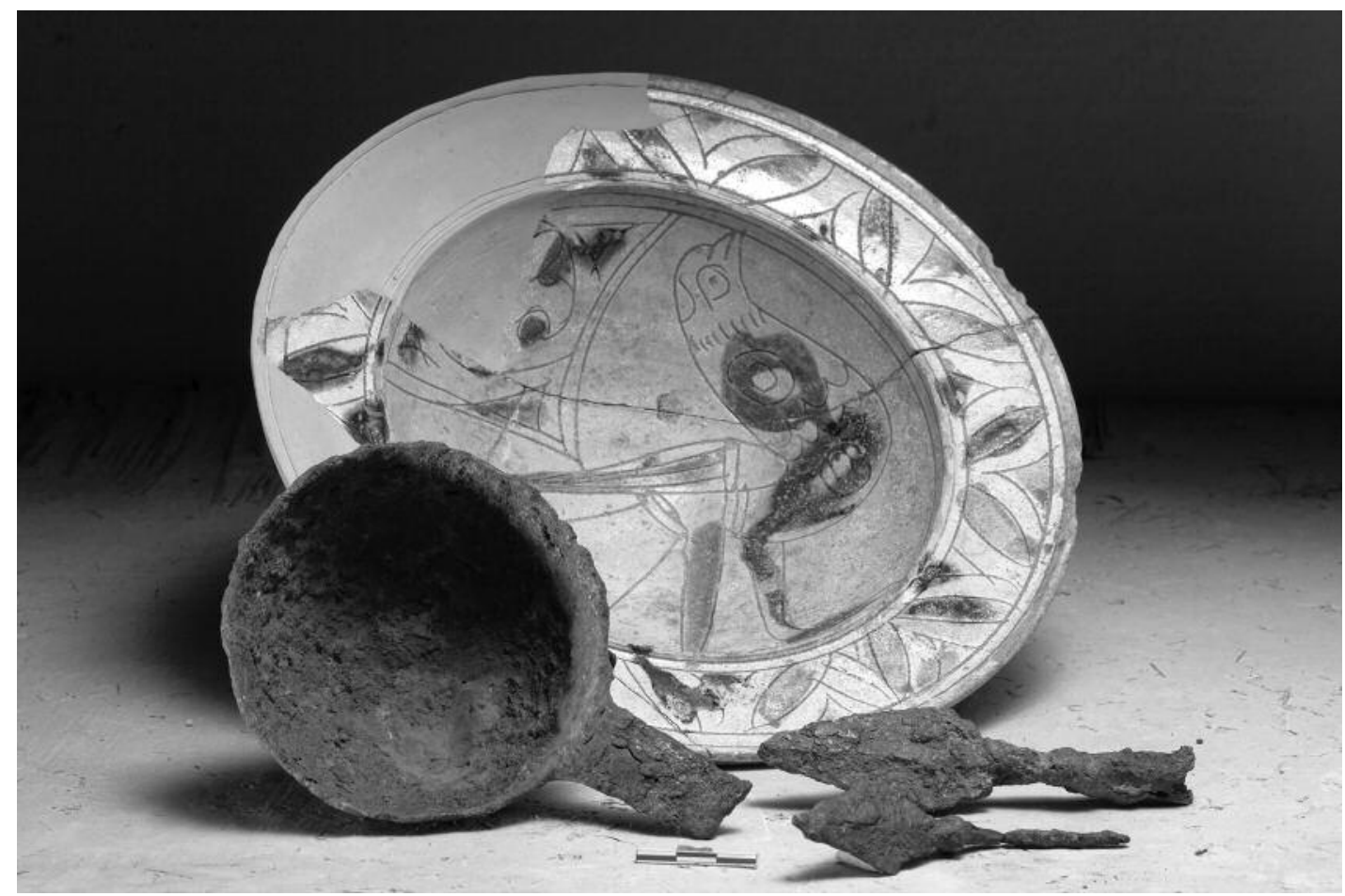

Fig. 22 : Vaisselle et armes médiévales. 
exemple, les tombes des phases du Néolithique Ancien et Moyen, jamais mises au jour dans les fouilles, ni, d'ailleurs, dans les chantiers de construction de la ville qui s'est développée tout autour du site ? A l'époque Chalcolithique, combien de portes y avaitil dans la citadelle ? Comment l'espace central étaitil organisé ? Quel était le rapport chronologique et fonctionnel entre les deux parties de la fortification et les maisons en terrasses ? Ensuite, le grand bâtiment des niveaux XV-XIII était-il seul et devrait-il donc être considéré comme "palais" ? Ou bien y en avait-il d'autres, auquel cas s'agissait-il plutôt d'un système de fonctionnement domestique répandu dans le village qu'ils mettaient en évidence? En ce qui concerne le deuxième millénaire, mis à part quelques rares tessons, où sont les vestiges du Bronze Moyen ? Où sont les restes du grand mur de "fortification hittite" découvert par Garstang près du sommet ? Le Fer Ancien est-il véritablement absent de Yumuktepe ? Et puis, où se trouve l'habitat de l'âge du Fer ? Enfin, où sont les maisons médiévales qui font référence au grand nombre de vases greniers stockés sur les pentes de la colline?

Après les "où", les "comment" et les "est-ce que" viennent les "pourquoi" et les "qui", auxquels il est encore plus difficile de répondre, à commencer par : pourquoi une citadelle au Chalcolithique Moyen ? Qui habitait les petites maisons ? Pourquoi les fours étaient-ils si nombreux dans la phase des "modifications" de la citadelle ? Qui étaient les habitants du grand bâtiment, pour lequel un investissement impressionnant dans le travail fut nécessaire? A qui la nourriture était-elle distribuée avec les centaines de bols retrouvés dans l'édifice ? Pourquoi un tel site fut-il abandonné pendant tout le millénaire suivant ? Pourquoi n'y a-t-il aucune trace de la culture Uruk, par ailleurs si répandue en Anatolie sud orientale ? Pourquoi aucune architecture de l'âge du Fer n'a-t-elle été retrouvée, alors que le répertoire céramique de ses couches à Yumuktepe est très riche et de grande qualité ? De manière plus générale, quel fut le rôle "politique" de ce site à chaque période de sa longue occupation?
Ces questions sont importantes et ce n'est que sur la base des réponses apportées qu'un cadre cohérent de l'histoire ancienne de la région de Mersin pourra être proposé. Le fait de circonscrire et de définir les interrogations peut déjà indiquer quelques voies à suivre dans la recherche. Ce seront de toute façon les fouilles et les analyses à venir qui apporteront les meilleures réponses. Sans aucun doute en créant de nouvelles questions, pour les nouvelles générations de chercheurs...

\section{REMERCIEMENTS}

Nos remerciements vont à la Direction générale des Biens culturels et des Musées du Ministère de la Culture et du Tourisme de Turquie pour l'autorisation accordée chaque année aux fouilles soutenues financièrement et menées au nom de l'Université del Salento. Pour le bon déroulement de la campagne 2015, nous exprimons toute notre reconnaissance au Ministère des Affaires étrangères d'Italie, à la Mairie de Toroslar, à la Chambre de Commerce et d'Industrie de Mersin, à la Bourse de Commerce de Mersin et, tout particulièrement, à la Mairie de Mersin. Nous remercions aussi l'ensemble de l'équipe de fouille et les ouvriers pour les efforts menés durant toute la durée de la mission, ainsi que, pour leur soutien, le représentant du ministère et la direction du Musée archéologique de Mersin.

\section{L'équipe de la saison 2015}

Silvia Ficco, Giulio Palumbi, Ernesto Santucci, Orkun Hamza Kayıcı et Ozan Konay, préhistoriens, Vincent Blanchard, spécialiste des âges du Bronze et du Fer, Tülay Özaydın, spécialiste de l'âge du Fer, Gülgün Köroğlu, spécialiste du Moyen-Age, Luigi Cinque et Emanuela Brunacci, topographes, Anna Maria Graziani, restauratrice, Burhan Ulaş, archéobotaniste, Claudia Minniti, archéozoologue, Y1lmaz Erdal, anthropologue, Alice Siracusano, dessinatrice, Hüseyin Tozun, Lenan Pinal et Melike Tunay, étudiants, et Özkan Tosun, représentant du ministère.

I.C. et E.J.

\section{BIBLIOGRAPHIE}

Breniquet, C., 1995 : "La stratigraphie des niveaux préhistoriques de Mersin et l'évolution culturelle en Cilicie", Anatolia Antiqua III : 1-31.

Brice, W., 2004 : "Preface", in Caneva, I. et Sevin, V. (éds.), Mersin-Yumuktepe. A Reappraisal, Congedo ed. Università del Salento, Galatina : 7.

Caneva, I., 2004a : "The early human occupation (7000-6000 BC)", in Caneva, I. et Sevin, V. (éds.), Mer-
sin-Yumuktepe. A Reappraisal, Congedo ed. Università del Salento, Galatina : 33-44.

$-2004 \mathrm{~b}$ : "Of terraces, silos and ramparts (60005800 BC)", in Caneva, I. et Sevin, V. (éds.), Mersin-Yumuktepe. A Reappraisal, Congedo ed. Università del Salento, Galatina : 45-56.

$-2004 \mathrm{c}$ : "The citadel tradition (5000-4200 BC)", in Caneva, I. et Sevin, V. (éds.), Mersin-Yumuktepe. A Reap- 
praisal, Congedo ed. Università del Salento, Galatina : 57-72.

- 2010a : "The History of the Landscape and Settlement", in Caneva, I. et Köroğlu, G. (éds.), A journey through nine thousand years, Ege Yayınları, Istanbul : 33-44.

- 2010b : "The Early Prehistoric Occupation: The Neolithic (7000-5800 BC)", in Caneva, I. et Köroğlu, G. (éds.), A journey through nine thousand years, Ege Yayınlar1, Istanbul : 17-36.

- 2010c : "The Late Prehistoric Occupation : The Chalcolithic Age (5000-4200 BC)", in Caneva, I. et Köroğlu, G. (éds.), A journey through nine thousand years, Ege Yayınları, Istanbul : 37-50.

-2012 : "Mersin-Yumuktepe in the Seventh Millennium BC: an updated view", in Özdoğan, M., Başgelen, N. et Kuniholm, P. (éds.), The Neolithic in Turkey, vol. 3: Central Turkey, Istanbul, Archaeology and Art Publications : $1-29$.

- sous presse : "'Mersin-Yumuktepe in the Seventh Millennium BC: the Social Dimension of Technological Changes", in Biehl, P. et Rosenstock, E. (éds.), Times of Change : 6000 BC in the Near East and Europe, Cambridge University Press.

Caneva, I. et Köroğlu, G. (éds.), 2010 : Yumuktepe. A journey through nine thousand years, Ege Yayınlar1, Istanbul.

Caneva, I. et Marcolongo, B., 2004 : "The history of the mound", in Caneva, I. et Sevin, V. (éds.), Mersin-Yumuktepe. A Reappraisal, Congedo ed. Università del Salento, Galatina : 9-16.

Caneva, I., Palumbi, G. et Pasquino, A., 2012 : "The Ubaid Impact on the Periphery: Mersin-Yumuktepe during the Fifth Millennium BC", in Marro, C. (éd.), After the Ubaid: interpreting changes from the Caucasus to Mesopotamia at the down of urban civilisation (4500-3500 $B C)$. Varia Anatolica XXVII, IFEA, De Boccard : 353389.

Caneva, I. et Sevin, V., 2004 : Mersin-Yumuktepe. A Reappraisal, Congedo ed. Università del Salento, Galatina.

Garstang, J., 1937 : "Explorations in Cilicia. The Neilson Expedition: Preliminary Report", Annals of Archaeology and Anthropology XXIV/1-2 : 52-68, pl. XIXIX.

- 1953 : Prehistoric Mersin. London.

Goldman, H., 1956 : Excavations at Gözlü Kule Tarsus, II. From the Neolithic through the Bronze Age, Princeton.

Jean, E., 2006 : "The Hittites at Mersin-Yumuktepe: Old Problems and New Directions", in Mielke, D.P., Schoop, U.D. et Seeher, J. (éds.), Structuring and Dating in Hittite Archaeology. Requirements, Problems, New Approaches, BYZAS 4, Istanbul : 311-332.

- 2010 : Sociétés et pouvoirs en Cilicie au $2^{e}$ millénaire av. J.-C. : approche archéologique, Thèse de Doctorat non publiée.
Köroğlu, G., 2004 : "Yumuktepe in the Middle Ages", in Caneva, I. et Sevin, V. (éds.), Mersin-Yumuktepe. A Reappraisal, Congedo ed. Università del Salento, Galatina : 103-132.

- 2010 : "Yumuktepe in the Middle Ages", in Caneva, I. et Köroğlu, G. (éds.), A journey through nine thousand years, Ege Yayınları, Istanbul : 79-104.

Köroğlu, K. (éd.), 1998 : 5. Yllında Yumuktepe. The V. Anniversary of the Excavations at Yumuktepe (19931997), Istanbul.

Köroğlu, K., 2010 : “A Hittite Stronghold on the Mediterranean Coast: Yumuktepe", in Caneva, I. et Köroğlu, G. (éds.), A journey through nine thousand years, Ege Yayınlar1, Istanbul : 65-72.

Kozal, E., 2015 : "A Discussion of the Origin and the Distribution Patterns of Red Lustrous Wheel-Made Ware in Anatolia : Cultural Connections Across the Taurus and Amanus Mountains", in Beyer, D., Henry, O. et Tibet, A. (éds.), La Cappadoce méridionale de la préhistoire à la période byzantine, $3^{\text {èmes }}$ Rencontres d'archéologie de l'IFEA, Istanbul 8-9 novembre 2012, Istanbul : 53-64.

Manuelli, F., 2009 : " Local Imitations and Foreign Imported Goods. Some problems and new questions on Red Lustrous Wheel-made Ware in the light of new excavations of the Southern Steep Trench at YumuktepeMersin", Altorientalische Forschungen 36/2 : 251-267.

- 2010 : "The Late Bronze Age", in Caneva, I. et Köroğlu, G. (éds.), A journey through nine thousand years, Ege Yayınları, Istanbul : 57-64.

Mellink, M.J., 1989: "Anatolian and Foreign Relations of Tarsus in the Early Bronze Age", in Emre, K. et al. (éds.), Anatolia and the Ancient Near East. Studies in Honor of Tahsin Özgüç, Ankara : 319-31.

Özaydin, T., 2010 : "Iron Age Strata”, in Caneva, I. et Köroğlu, G. (éds.), A journey through nine thousand years, Ege Yayınları, Istanbul : 73-78.

Palumbi, G., 2010 : "The Early Bronze Age", in Caneva, I. et Köroğlu, G. (éds.), A journey through nine thousand years, Ege Yayınlar1, Istanbul : 51-56.

Sevin, V., 2004 : "Introduction", in Caneva, I. et Sevin, V. (éds.), Mersin-Yumuktepe. A Reappraisal, Congedo ed. Università del Salento, Galatina : 13-19.

Sevin, V. et Caneva, I., 1998 : "Recent Excavations on Mersin/Yumuktepe: A New Prelude", in Köroğlu, K. (éd.), 5. Yılında Yumuktepe. The V. Anniversary of the Excavations at Yumuktepe (1993-1997), Istanbul : 5-7.

Sevin, V. et Köroğlu, K., 2004 : "Late Bronze Age at Yumuktepe: new evidence from Step-Trench South", in Caneva, I. et Sevin, V. (éds.), Mersin-Yumuktepe. A Reappraisal, Congedo ed. Università del Salento, Galatina : 73-83.

Sevin, V. et Özaydın, T., 2004 : “'Iron Age levels”, in Caneva, I. et Sevin, V. (eds.), Mersin-Yumuktepe. A Reappraisal, Congedo ed. Università del Salento, Galatina : 85-101.

Ulaş B., 2014 : "Mersin Yumuktepe Höyüğü Arkeobotanik Çalışmaları”, ANMED 2014-12 : 306-31. 


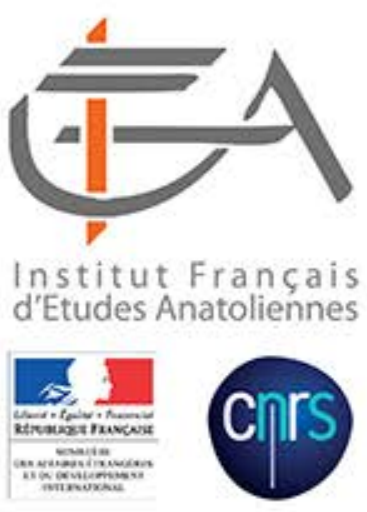

Eurômos : le temple de Zeus vu de l'Est (cliché Sönmez Alemdar).

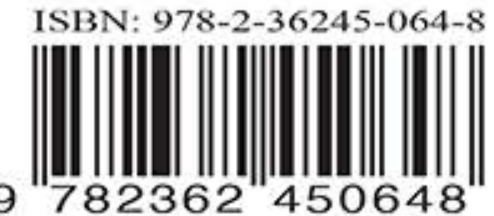

